\title{
6. Wahrheit
}

Die bisherigen Überlegungen galten der Analyse dessen, was wir tun, wenn wir die Überzeugungs- und Rechtfertigungsbedingungen für Wissen erfüllen. Es hat sich gezeigt, dass beide Bedingungen Können voraussetzen, weshalb das Gleiche letztlich auch für Wissen gilt. In diesem Kapitel wird es schließlich um die Wahrheitsbedingung gehen. Ich werde geltend machen, dass das zuvor entwickelte Erklärungsmodell für das Verhältnis von Wissen und Können durch sie letztlich nicht beeinflusst wird. Die Frage ist, ob es eine substantielle Theorie gibt, die Wahrheit über die bisherige, normative Charakterisierung hinaus erklären kann. Um zu zeigen, dass dem nicht so ist, werde ich zunächst einen Überblick über einige wichtige Wahrheitstheorien geben und deren Vor- und Nachteile diskutieren. Ich werde geltend machen, dass ein deflationistisches Verständnis dazu geeignet ist, all die Probleme zu vermeiden, mit denen die anderen Theorien konfrontiert sind. Demnach ist Wahrheit ein einzigartiger logischer Begriff, der eine expressive und generalisierende Funktion erfüllt. Daraus ergibt sich zugleich eine Antwort auf die Frage nach seiner epistemologischen Funktion. Es wird sich zeigen, dass Wahrheit auch für die Wissensanalyse rein expressive Bedeutung hat. Daher ist sie epistemologisch gesehen irrelevant. Das eigentliche Thema der Arbeit, eine anti-intellektualistische Fundierung von Wissen durch Können, bleibt vom Wahrheitsbegriff letztlich unbeeinflusst.

\subsection{Wahrheit aus Sicht einer normativen Pragmatik}

Um jemanden als Wissenden bezeichnen zu können, muss man ihm zunächst die Festlegung auf eine bestimmte Überzeugung zuschreiben. Behauptet jemand beispielsweise, dass am Freitag ein Feiertag ist, so können wir ihn als eben darauf festgelegt betrachten. Mit dieser Festlegung verbinden sich weitere Festlegungen und Berechtigungen. Wer etwa glaubt, dass am Freitag ein Feiertag ist, ist auch darauf festgelegt, dass es einen Unterschied gibt zwischen Feiertagen und gewöhnlichen Werktagen. Ebenso ist er zu weiteren Behauptungen berechtigt wie etwa der, dass am Freitag die Geschäfte geschlossen sind. Eine Festlegung kann also dazu 
dienen, eine andere zu plausibilisieren und zu stützen. Im Prinzip ist eine Behauptung immer auch eine Rechfertigung.

Als Behauptungen sind Überzeugungen Teil der Praxis des Gebens und Verlangens von Gründen. Mit einer Festlegung verbindet sich die Frage, welche Gründe es dafür gibt und wofür sie selbst als Grund dient. Im Netz der Gründe konstituiert sich einerseits Bedeutung. Andererseits liegt darin die Möglichkeit, eine Behauptung durch eine weitere $\mathrm{zu}$ rechtfertigen. Eine Behauptung ist niemals eine einzelne, isolierte Aussage. Sie ist immer durch andere Behauptungen begründet und Grund für weitere Behauptungen. Das ist der Kerngedanke der inferentiellen Semantik. Ein und dieselbe inferentielle Struktur dient einerseits der Konstitution von Bedeutung und andererseits der Rechtfertigung von Behauptungen. Ein Vorteil dieses Erklärungsansatzes ist der, dass er das Auftreten eines Rechtfertigungsregresses vermeidet. Schließlich müssen einige Inferenzen als (material) korrekt vorausgesetzt werden, wenn ein sinnvoller und bedeutsamer Gebrauch von Sprache überhaupt möglich sein soll. Nur unter dieser Voraussetzung können in einem zweiten Schritt Rechtfertigungen eingefordert werden. ${ }^{270}$

Die Rekonstruktion der Überzeugungs- und der Rechtfertigungsbedingung ist mit Hilfe des normativ-inferentiellen Theoriemodells der vorausgehenden Kapitel sehr gut möglich. Wie verhält es sich nun mit der Wahrheitsbedingung? Bisher ist Wahrheit nur als Anspruch oder normativer Standard aufgetreten. Ich habe sie als Teil der normativen Signifikanz des Sprachspiels der Überzeugung beschrieben: Behauptungen werden mit dem Anspruch, wahr zu sein, gemacht oder zugeschrieben. Was jemand behauptet, soll wahr sein, zumindest in der überwiegenden Zahl der Fälle. Eine Person allein kann diesen Anspruch aber nicht erfüllen. Sie kann zwar der Forderung nachkommen, ihre Behauptung zu rechtfertigen. Zu diesem Zweck kann sie weitere Überzeugungen anführen. So kann sie feststellen, dass ihre Behauptung gerechtfertigt ist. Dass sie wahr ist, können allerdings nur andere bestätigen.

Mehr noch als für die Bedingung der Überzeugung und der Rechtfertigung ist die der Wahrheit auf die soziale Perspektive der

${ }^{270}$ Vgl. ebd., 305 f. 
sprachlichen Praxis angewiesen. ${ }^{271}$ Aus Sicht der normativen Pragmatik betrachtet und behandelt jemand die Behauptung eines anderen als wahr, indem auch er sie sich zu eigen macht. Sage ich beispielsweise: „Es ist wahr, dass am Freitag ein Feiertag ist.", so lege ich mich selbst darauf fest, dass am Freitag ein Feiertag ist. Dass ich dies als wahr bezeichne, signalisiert, dass ich damit eine Behauptung übernehme, die bereits im Gespräch ist. Offensichtlich kommt es also mit der Rede von Wahrheit zu einer Wiederholung oder Übertragung des normativen Status der Festlegung. Wer die Festlegung eines anderen Diskursteilnehmers als wahr betrachtet, schreibt sie ihm nicht nur zu, er geht sie selber ein. Jemanden als auf eine wahre Überzeugung festgelegt zu betrachten, heißt dann, sich selbst darauf festzulegen.

Dies ist eine noch recht vage Bestimmung der Wahrheitsbedingung von Wissen. Entsprechend vage fällt die Rekonstruktion der Standarddefinition von Wissen aus. Demnach betrachten wir jemanden als Wissenden, wenn wir ihm eine Festlegung und eine Berechtigung zuschreiben und wenn wir dieselbe Festlegung selbst eingehen. ${ }^{272}$ Anders gesagt weiß jemand, dass $p$, wenn er sich darauf festlegt, dass $p$, wenn er dazu berechtigt ist, sich darauf festzulegen, dass $p$, und wenn es wahr ist, dass $p$. Insbesondere der Teil, der die Wahrheitsbedingung zum Ausdruck bringt, bedarf hier weiterer Erläuterungen, soll er als Ausschlusskriterium dienen können. Im Folgenden werde ich daher einige wichtige Wahrheitstheorien diskutieren mit dem Ziel, eine geeignete Ergänzung für die bisherige Rekonstruktion von Wissen zu finden. Vom Erfolg dieser Suche hängt $a b$, ob und in welcher Form dieses Kriterium beibehalten werden kann.

\subsection{Korrespondenztheorie}

Die meisten Menschen werden intuitiv der Behauptung zustimmen, dass Wahrheit etwas mit der Übereinstimmung unserer Überzeugungen oder Aussagen mit der Wirklichkeit zu tun hat. Damit befürworten sie bewusst oder unbewusst, was in der Philosophie als Korrespondenztheorie

\footnotetext{
${ }^{271}$ Vgl. Davidson (1984), 170.

${ }^{272}$ Vgl. Brandom (1994), 202.
} 
der Wahrheit bezeichnet wird. Die Zahl der Autoren, die eine Version dieser Theorie vertreten ist groß. ${ }^{273}$ Berühmt ist Aristoteles' Formel: „Zu sagen nämlich, das Seiende sei nicht oder das Nicht-Seiende sei, ist falsch, dagegen zu sagen, das Seiende sei und das Nicht-Seiende sei nicht, ist wahr. ${ }^{\text {2074 }}$ Eine andere bekannte Formulierung findet sich bei Thomas von Aquin: „Veritas est adaequatio rei et intellectus (...) Wahrheit ist die Angleichung eines Dinges und des Verstandes.“275 Eine mittlerweile klassische Formulierung aus der jüngeren Vergangenheit stammt von Bertrand Russell: „Thus a belief is true when there is a corresponding fact, and is false when there is no corresponding fact. “276

Gemein ist all diesen Konzeptionen die Idee, dass Wahrheit in der Relation zur Wirklichkeit besteht. Dies gilt als ultimativer Test, mag es auch Überzeugungen oder Aussagen geben, die sich nicht unmittelbar auf diese Weise verifizieren lasse. Von Russell und George E. Moore stammt die einflussreiche Modifikation, Überzeugungen oder Aussagen nicht mehr auf konkrete oder abstrakte Gegenstände, sondern auf Tatsachen zu beziehen. ${ }^{277}$ Demnach ist eine Überzeugung wie beispielsweise die, dass eine Katze auf der Matte liegt, deswegen wahr, weil es in der Welt eine Tatsache gibt, die sich aus einer Katze, einer Matte und der Eigenschaft des Liegens zusammensetzt. Die unterschiedlichen Vertreter der Korrespondenztheorie glauben jedenfalls, dass mit Hilfe einer präzisen Vorstellung dessen, was unter Korrespondenz, Wirklichkeit, Tatsachen etc. $\mathrm{zu}$ verstehen ist, eine aufschlussreiche Wahrheitstheorie möglich ist. Sie schätzen an dieser Konzeption, dass sie starken Intuitionen folgt und weitestgehend ohne umstrittene Ideen auskommt. Als ihr großer Vorteil

273 Zu der Vermutung, dass vor Kant alle Philosophen Vertreter einer Korrespondenztheorie der Wahrheit waren, vgl. Putnam (1981), 56. Zu einem Überblick vgl. auch David (2004), Kap. 3.

${ }^{274}$ Aristoteles (1994), 1011b26. Vgl. Platon (1994c), 262e - 263d.

275 von Aquin (1986), Qu. 1, Art. 1, 8 f. T. von Aquin schreibt diese Formulierung I. Israeli zu.

${ }^{276}$ Russell (1982), 75.

277 Vgl. Moore (1953), Kap. 15, Russell (1982), Kap. 12, Russell (1906/07), Russell (1992), Teil 2, Kap. 5 u. Wittgenstein (1984e). 
gilt, dass sie lediglich das beinhaltet, was ohnehin offensichtlich ist. ${ }^{278}$ Tatsächlich führt jedoch die Vorstellung, Wahrheit lasse sich als eine Korrespondenzrelation bestimmen, schnell zu beträchtlichen Schwierigkeiten. Schuld daran sind einige zusätzliche Annahmen, die selbst wiederum alles andere als selbstverständlich sind. Hier sind nur drei Beispiele.

Erstens gehen die meisten Korrespondenztheoretiker davon aus, dass es eine von uns unabhängige Wirklichkeit oder Welt der Tatsachen gibt, auf die wir uns mit unseren Vorstellungen, Überzeugungen oder Aussagen beziehen. Diese Wirklichkeit oder Welt der Tatsachen ist größtenteils unabhängig davon, ob es uns oder irgendwelche Vorstellungen, Überzeugungen, Aussagen etc. gibt. Kurz gesagt: Sie existiert objektiv. Auch wenn wir verschwinden würden oder es uns nie gegeben hätte, wäre sie zum allergrößten Teil genau dieselbe. Mit der Korrespondenztheorie verbindet sich also ein metaphysischer Realismus bezüglich der Außenwelt oder der Welt der Tatsachen. ${ }^{279}$

Zweitens geht mit dieser Konzeption eine Unterscheidung zwischen Wahrheitsträgern wie Überzeugungen oder Aussagen auf der einen Seite und Wahrmachern wie Gegenständen oder Tatsachen auf der anderen einher. Damit sich nun Wahrheit in einem solchen Szenario feststellen lässt, muss sich die Korrespondenzrelation als ganze beurteilen lassen. Wahrheitsträger und Wahrmacher müssen sich zugleich oder zumindest in unmittelbarem Zusammenhang in den Blick nehmen lassen. Ob unsere Überzeugungen tatsächlich mit der Wirklichkeit übereinstimmen, lässt sich also nur dadurch überprüfen, dass wir sie und damit unsere eigene, involvierte Position transzendieren. Deshalb müssen Korrespondenztheoretiker für ihre Betrachtung die Möglichkeit eines externalistischen

${ }^{278}$ Vgl. z. B. Kant (1998), B82 (A58), James (1991), 87 o. Searle (1995), 189, wobei die allgemeinen Positionen Kants und James' jedoch keine Korrespondenztheorie der Wahrheit beinhalten.

${ }^{279}$ Vgl. Dummett (1958/59), 157, Putnam (1978), 18. o. Rorty (1991), 22. Zu der Auffassung, dass die Korrespondenztheorie auch zusammen mit einem Antirealismus vertreten werden kann, üblicherweise aber einen Realismus impliziert, vgl. David (2004), 370 u. Searle (1995), 154. 
Standpunktes voraussetzen. ${ }^{280}$ Dieser befindet sich außerhalb unserer eigenen Vorstellungen, Überzeugungen und Aussagen. Von ihm aus soll sich überprüfen lassen, ob das, was wir in Bezug auf die Welt glauben und sagen, mit dem korrespondiert, wie die Welt als solche beschaffen ist.

Drittens beinhaltet die Korrespondenztheorie die Annahme, dass jedem Gegenstand oder jeder Tatsache in der Wirklichkeit eine bestimmte Vorstellung oder Überzeugung entspricht. Dieses Entsprechungsverhältnis kann zwar auf beiden Seiten komplexe Strukturen annehmen. Gegenstände oder Tatsachen können sich aus verschiedenen Bestandteilen, Eigenschaften oder Relationen zusammensetzen. Ebenso können sich Überzeugungen oder Aussagen aus Begriffen, Ausdrücken oder Wörtern zusammensetzen. Nach dem einfachsten Verständnis bedeutet Korrespondenz jedoch eine Art Eins-zu-eins-Relation zwischen Wahrheitsträger und Wahrmacher. Eine Korrespondenzrelation besteht also genau dann, wenn einer Tatsache wie etwa der, dass die Katze auf der Matte liegt, eine gleichermaßen strukturierte Überzeugung oder Aussage entspricht.

\subsection{Transzendenz- und Referenzproblem}

Es sind vor allem zwei Problemkomplexe, die sich aus den Zusatzannahmen der Korrespondenztheorie ergeben. Ich will sie das Transzendenzproblem und das Referenzproblem nennen. Ersteres lässt sich auf die recht einfache Frage reduzieren: Wie lässt sich eine Korrespondenzrelation eigentlich erkennen oder überprüfen? Sie zielt darauf ab, dass Korrespondenztheoretiker einer systematischen Unterscheidung von Überzeugungen und ihrem Abgleich mit der Wirklichkeit kaum Beachtung schenken. Die einzig mögliche Antwort besteht nämlich in dem, was die zweite der erwähnten Zusatzannahme beinhaltet: der Einnahme eines externalistischen Standpunktes. Wenn wir feststellen wollen, ob unsere Überzeugungen oder Aussagen mit der von uns unabhängigen Wirklichkeit übereinstimmen, dann müssen wir einen

${ }^{280}$ Vgl. z. B. Nagel (1986), 68 ff. Die Idee eines solchen Standpunktes geht natürlich auf Descartes' Verfahren in den Meditationen zurück. Vgl. Descartes (1964). Zur Unterscheidung zwischen einem externalistischen und einem internalistischen Standpunkt vgl. Putnam (1981), Kap. 3. 
Standpunkt einnehmen, von dem aus sich beide Bereiche zugleich einsehen und überprüfen lassen. Wir müssen - zumindest für den Moment - all unsere Beteiligung einstellen und die Korrespondenzrelation selbst in den Blick nehmen. Anders ist eine Beurteilung nicht möglich.

Das Problem ist aber, dass es einen solchen unbeteiligten Standpunkt sinnvollerweise gar nicht geben kann. Es ist offensichtlich, dass wir nicht aus uns selbst heraustreten können, um einen unabhängigen Blick auf unsere Überzeugungen oder Aussagen und die Wirklichkeit zu werfen. Es gibt keinen archimedischen Punkt, von dem aus sich beurteilen ließe, welche Überzeugungen oder Aussagen maßgeblich sind und welche nicht. ${ }^{281}$ Wäre es möglich, den eigenen Standpunkt auf eine solche Weise zu transzendieren, dann würde sich die Frage nach der Wahrheit von selbst erledigen. Für einen externen Betrachter lägen die Dinge dann bereits so vor, wie sie (wahrheitsgemäß) sind. Dies ist aber offensichtlich nicht der Fall. Stattdessen führt jeder Versuch, unsere Überzeugungen oder Aussagen mit der Wirklichkeit abzugleichen, immer nur zu weiteren Überzeugungen oder Aussagen. ${ }^{282}$ Ein Heraustreten aus unseren Überzeugungen oder unserer Sprache ist also überhaupt nicht möglich.

Das Transzendenzproblem lässt sich noch weiter zuspitzen. Wir können nämlich nicht nur fragen, wie sich die Korrespondenzrelation erkennen und überprüfen lässt, sondern ob dies überhaupt möglich ist. Wir können an dieser Relation als solcher zweifeln. Die Vorstellung, es gebe eine von uns unabhängige Wirklichkeit und es sei möglich, unsere Situation darin zu transzendieren, wirft die Frage auf, ob wir überhaupt einen verifizierbaren Zugang $\mathrm{zu}$ dieser Wirklichkeit haben. Dies ist bekanntlich die Frage des Skeptikers. Das Problem ist also, dass die Korrespondenztheorie mit ihren realistischen Implikationen unmittelbar zu einem Außenweltskeptizismus einlädt. ${ }^{283}$ Der Realismus schafft Raum für Zweifel nicht nur daran, dass wir mit einigen unserer Überzeugungen und

${ }^{281}$ Zur Annahme, dass es zumindest teilweise möglich ist, den gegenwärtigen Standpunkt zu transzendieren, um sich einem objektiven Bild der Wirklichkeit anzunähern, vgl. Nagel (1986), insbes. Kap. 5. T. Nagel ist bereit, für diese Annahme einen Skeptizismus der Außenwelt in Kauf zu nehmen.

${ }^{282}$ Vgl. Davidson (1990), 302.

${ }^{283}$ Vgl. Putnam (1981), 50 o. Nagel (1986), 70 f. u. 90. 
Aussagen in Bezug auf die Außenwelt richtig liegen, sondern dass dies jemals so ist.

Dies erklärt sich durch die Hierarchisierung der Überzeugungen und der entsprechenden Aussagen, die damit einhergeht. ${ }^{284}$ Denn indem der Korrespondenztheoretiker die Relation zur Wirklichkeit zum ultimativen Test für Wahrheit macht, hält er stillschweigend eine bestimmte Klasse von Überzeugungen im Vergleich zu allen anderen für grundlegend. Das sind Überzeugungen, die das Resultat von Erfahrungen sind. Er ignoriert dabei, dass zwischen Überzeugungen darüber, wie die Welt erscheint, und solchen darüber, wie die Welt ist, kein gültiger Begründungszusammenhang besteht. Träume, Illusionen und Sinnestäuschungen weisen darauf hin, dass unsere Erfahrung keine uneingeschränkt zuverlässige Informationsquelle ist, was die wirkliche Beschaffenheit der Welt angeht. Daher die Hartnäckigkeit, mit der der Skeptiker seine Frage zu stellen vermag. Erfahrung und Zweifel gehen Hand in Hand. Wer also gerade diese Art von Überzeugungen zu einer privilegierten Klasse erklärt, setzt das gesamte System unserer Überzeugungen von Anfang an einem globalen Zweifel aus.

Der Korrespondenztheoretiker kann diese Kritik als eine Fehlinterpretation abtun. Er kann geltend machen, dass es sich bei der Übereinstimmung mit der Wirklichkeit oder mit den Tatsachen gerade um eine nicht-epistemische Relation handelt. ${ }^{285}$ Seine Theorie dürfe daher nicht als eine Antwort auf die Frage verstanden werden, wie wir herausfinden können, ob eine Überzeugung oder Aussage wahr oder falsch ist. Sie erhebe gar nicht den Anspruch zu erklären, wie es kommt, dass Überzeugungen oder Aussagen mit realen Gegenständen oder Tatsachen korrespondieren. Sie gehe lediglich davon aus, dass dies prinzipiell möglich ist. Andernfalls müssten all unsere Überzeugungen oder Aussagen über die Außenwelt als sinnlos erscheinen. So verstanden beansprucht die Korrespondenztheorie gar nicht die Möglichkeit, dass wir aus unseren Überzeugungen oder unserer Sprache heraustreten. Der Vorwurf, diese

${ }^{284} \mathrm{Zu}$ der Auffassung, dass Realismus und Externalismus deshalb unmittelbar zum Außenweltskeptizismus führen, weil sie einen epistemologischen Fundamentalismus voraussetzen, vgl. auch Williams (1986), insbes. $418 \mathrm{ff}$.

${ }^{285}$ Vgl. Davidson (1990), $302 \mathrm{f}$. 
Konzeption provoziere einen Außenweltskeptizismus, erscheint also als ungerechtfertigt.

Das Referenzproblem zeigt aber, dass diese Replik nicht zur Plausibilität der Korrespondenztheorie beitragen kann. Denn die Korrespondenzrelation ist auch dann problematisch, wenn wir sie als eine nicht-epistemische verstehen. $\mathrm{Ob}$ und wie sich diese Relation erkennen lässt, ist nur die eine Frage. Ob und wie sich deren Relata bestimmen lassen, ist die andere. Damit ist insbesondere das gemeint, was unter den Begriff des Wahrmachers fällt. Dieser Teil der Relation ist nämlich bestenfalls unbestimmt, wenn nicht gar eine Chimäre. ${ }^{286}$ Unklar ist, ob sich das, womit Aussagen oder Sätze korrespondieren sollen, überhaupt unzweideutig identifizieren lässt. Die Kritik gilt dabei vor allem der Annahme, bei der Korrespondenzrelation handle es sich um eine Eins-zueins-Relation zwischen Wahrheitsträger und Wahrmacher. Damit erklärt der Korrespondenztheoretiker Wahrheit nämlich zu einer Angelegenheit der direkten Bezugnahme von Wörtern oder Sätzen auf Gegenstände, Sachverhalte oder Tatsachen. Und genau hier liegt das Problem.

Das Referenzproblem war bereits Thema im Zusammenhang der Diskussion repräsentationalistischer Bedeutungstheorien. ${ }^{287}$ Dort hat sich am Beispiel der hinweisenden Definition gezeigt, dass die Bezugnahme von Ausdrücken auf Gegenstände keineswegs gesichert und eindeutig ist. Dies lässt sich auf den Unterschied zwischen Referenz und Bedeutung zurückführen. Dieses Problem diagnostiziert auch Quine mit seiner These von der Unbestimmbarkeit von Übersetzung und Referenz. ${ }^{288}$ Demnach gibt es für jeden Gegenstand oder Sachverhalt prinzipiell mehrere Möglichkeiten der sprachlichen Bezugnahme. Eindeutige Anhaltspunkte, welcher von ihnen der maßgebliche ist, gibt es nicht. Wenn sich also keine

286 Zur Auffassung, dass Sätze oder Aussagen - wenn sie überhaupt mit etwas korrespondieren - alle mit ein und demselben korrespondieren (der ,großen Tatsache“ oder dem Universum), vgl. Davidson (1984), 18 f. u. 41 f. u. Davidson (1990), 303 f. Davidson greift auf das sogenannte Slingshot-Argument zurück, das ursprünglich auf Frege und A. Church zurückgeht. Vgl. Frege (1994), 49 f. u Church (1956), 24 f. Vgl. auch Lewis (1946), 50-55 u. Wallace (1969). Die Gültigkeit dieses Arguments ist umstritten. Vgl. z. B. Searle (1995), 221 ff. o. Neale (2001), 49 ff.

${ }^{287}$ Vgl. erneut Kap. 5.3.

${ }^{288}$ Vgl. erneut Anm. 126. 
Tatsache als diejenige aussondern lässt, auf die sich ein Ausdruck oder Satz bezieht, dann kann man sie ebenso gut ganz verneinen: ,[T]here is no fact of the matter. “289

Übertragen auf die Korrespondenztheorie der Wahrheit heißt das, dass Sätze oder Aussagen - wenn überhaupt - mit unterschiedlichen Gegenständen oder Tatsachen in Relation stehen. Umgekehrt können wir uns mit unterschiedlichen Ausdrücken oder Aussagen auch auf ein und denselben Gegenstand oder ein und dieselbe Tatsache beziehen. Jedenfalls ist die (vermeintliche) Bezugnahme von Wahrheitsträgern auf Wahrmacher prinzipiell unbestimmt. Es gibt keine Möglichkeit, die für die Bestimmung von Wahrheit relevante Korrespondenzrelation verlässlich auszusondern. Das Referenzproblem beinhaltet also weniger die Einsicht, dass es keine Korrespondenzen zwischen Sätzen und Tatsachen geben kann, sondern dass es davon $\mathrm{zu}$ viele gibt. ${ }^{290}$ Von Eins-zu-eins-Relationen zwischen Wahrheitsträgern und Wahrmachern kann also nicht die Rede sein. Nur so wären Korrespondenzrelationen aber intelligibel.

Wer also eine Korrespondenztheorie der Wahrheit vertritt, folgt zwar einer verbreiteten Intuition über den Zusammenhang von Sprache und Wirklichkeit. Wenn es aber um die Umsetzung dieser Konzeption geht, ist er mit einer Reihe von Problemen konfrontiert. Einer der Haupteinwände ist, dass wir nicht aus unseren Überzeugungen und unserer Sprache heraustreten können. Versuchen wir es dennoch, so generieren wir unweigerlich einen Skeptizismus der Außenwelt. Auch dass die Relata der Korrespondenzrelationen prinzipiell unbestimmt sind, spricht gegen diese Theorie. Worauf sich wahre Überzeugungen oder Aussagen beziehen, lässt sich nicht mit Gewissheit herausfinden. Wenn aber das Erkennen oder Individuieren von Korrespondenzrelationen fraglich ist, dann ist es letztlich die Korrespondenztheorie insgesamt. Als Wahrheitskriterium eignet sich Korrespondenz daher nicht.

${ }^{289}$ Quine (1969), 38 u. 47.

${ }^{290}$ Vgl. Putnam (1981), 72 f. 


\subsection{Kohärenztheorie}

Die realistischen Implikationen der Korrespondenztheorie fordern entweder das Heraustreten aus der Sprache oder die Annahme einer nichtepistemischen Korrespondenzbeziehung. Beides ist problematisch. Es gibt allerdings eine Alternative, die über die bisherigen Vorschläge hinausgeht. Sie besteht darin, bei der Bestimmung von Wahrheit innerhalb der Sprache und des Systems unserer Überzeugungen zu verbleiben und stattdessen die realistischen Intuitionen aufzugeben. Nicht die Übereinstimmung mit der Wirklichkeit ist dann das Kriterium für Wahrheit, sondern die Art und Weise, wie Aussagen oder Überzeugungen miteinander zusammenhängen. Gemäß dieser Konzeption gelten Aussagen oder Überzeugungen als wahr, wenn sie Teil eines kohärenten Systems sind. Dies ist die Idee der Kohärenztheorie der Wahrheit.

Autoren, die sich eindeutig als Vertreter dieser Theorie zu verstehen geben, sind vor dem 20. Jahrhundert schwer zu finden. ${ }^{291}$ Ihre Grundzüge formuliert beispielsweise Otto Neurath:

Aussagen werden mit Aussagen verglichen, nicht mit „Erlebnissen“, nicht mit einer „Welt“, noch mit sonst etwas. (...) Jede neue Aussage wird mit der Gesamtheit der vorhandenen, bereits miteinander in Einklang gebrachten, Aussagen konfrontiert. Richtig heißt eine Aussage dann, wenn man sie eingliedern kann. ${ }^{292}$

Der Vorteil dieses Verständnisses von Wahrheit ist, dass zwischen Wahrheitsträger und Wahrmacher nicht grundlegend unterschieden wird. Somit stellen sich auch keine skeptischen Fragen nach dem Realitätsbezug und seiner Erkennbarkeit. Die einzelne Aussage oder Überzeugung muss sich nicht im Test an der Wirklichkeit bewähren, sondern in dem kohärenten System, dessen Teil sie sein soll. Die Grundlage für die Bestimmung von Wahrheit ist somit ein Holismus in Bezug auf Überzeugungen.

291 Als Vorläufer werden häufig B. Spinoza, I. Kant, G. W. F. Hegel oder F. H. Bradley genannt. Gegenüber dieser Einordnung gibt es aber berechtigte Vorbehalte. Vgl. David (2004), 402 Anm. 42.

${ }^{292}$ Neurath (1931), 403. Vgl. auch Neurath (1932/33), 209. 
Was bedeutet in diesem Zusammenhang Kohärenz? Eine Minimalbedingung dafür, dass ein System von Aussagen oder Überzeugungen als kohärent bezeichnet werden kann, ist logische Konsistenz. ${ }^{293}$ Das heißt, dass die Aussagen oder Überzeugungen eines kohärenten Systems nicht im Widerspruch zueinander stehen dürfen. Dies ändert sich auch dann nicht, wenn dem System eine neue Aussage oder Überzeugung hinzugefügt wird. Darüber hinaus sollte das System reichhaltig sein und nicht auf einige wenige, wenn auch konsistente Überzeugungen beschränkt sein. Ebenso wenig sollte es sich auf eine einzige Art von Überzeugungen wie beispielsweise Wahrnehmungsüberzeugungen beschränken. Ein System, das zusätzlich Überzeugungen enthält, die beispielsweise der Erinnerung, der Introspektion oder der Abstraktion entstammen, ist reichhaltiger und in diesem Sinne auch kohärenter. Dann gehört zu einem kohärenten System, dass die darin enthaltenen Überzeugungen auch miteinander in Zusammenhang stehen. Eine Reihe einzelner Überzeugungen, die vollkommen unzusammenhängend ist, kann man nicht in demselben Maße als kohärent bezeichnen, wie eine, in der sich die Überzeugungen gegenseitig erklären, stützen oder wahrscheinlicher machen. Schließlich ist es für ein kohärentes System notwendig, dass es im Großen und Ganzen einfach ist. Um überprüfen zu können, dass eine neue Überzeugung nicht im Widerspruch $\mathrm{zu}$ den bereits vorhandenen steht, muss das System übersichtlich sein. Gleiches gilt für die Beurteilung der internen Zusammenhänge. In einem übermäßig komplexen und ausufernden System ist es nicht mehr möglich, diese überhaupt noch festzustellen.

Einige dieser Aspekte sind dem Leser bereits vertraut. Denn das kohärenztheoretische Verständnis von Wahrheit greift offensichtlich auf die gleiche systematische Infrastruktur zurück, wie zuvor die Überlegungen zu Bedeutung und Rechtfertigung. Man kann sagen, dass ein kohärentes System von Überzeugungen immer auch eines ist, in dem Überzeugungen inferentiell in Zusammenhang stehen. Die Kohärenztheorie lässt sich also als Ergänzung der inferentialistischen Festlegung verstehen, wonach nur Überzeugungen Gründe für weitere Überzeugungen sein können. Was für Bedeutung und Rechtfertigung der inferentielle Zusammenhang ist, ist für Wahrheit Kohärenz. Dies kann man zugleich als

${ }^{293} \mathrm{Vgl}$. auch für das Folgende Rescher (1973), 31 ff. u. Künne (2003), $383 \mathrm{ff}$. 
eine Präzisierung des dritten der erwähnten Kohärenzkriterien verstehen. Denn mit dem Zusammenhang kohärenter Überzeugungen ist offensichtlich gemeint, dass zwischen den Überzeugungen Inferenzbeziehungen formaler oder materialer Art bestehen. Ebenso betroffen ist davon das Kriterium der logischen Konsistenz. Schließlich gibt es gültige Schlüsse nur innerhalb eines widerspruchsfreien Systems von Überzeugungen.

Damit wird der Begriff der Wahrheit in die unmittelbare Nähe von Rechtfertigungs- oder Folgerungszusammenhängen gerückt. Diese Angleichung hat jedoch seine Grenzen. Denn es wäre verkehrt, hier eine allzu große Nähe oder gar Übereinstimmung zu sehen. Es mag ganz ähnliche Kriterien dafür geben, wann wir etwas als bedeutungsvoll bezeichnen und wann als wahr. Hinsichtlich der These, Wahrheit lasse sich auf die gleiche Weise auf inferentielle Zusammenhänge zurückführen wie Bedeutung, sind aber Zweifel angebracht. Dass nicht alles, was eine Bedeutung hat, zugleich auch wahr ist, wird kaum jemand bestreiten. Zwischen semantischer Korrektheit und Wahrheit oder Evidenz besteht zumindest in pragmatischer Hinsicht ein wichtiger Unterschied. ${ }^{294}$ Mit der Feststellung von Wahrheit verbindet sich ein ganz anderer Anspruch als mit der von Bedeutung. Wer etwas als wahr einschätzt oder bezeichnet, hat andere Erwartungen, als der, der etwas versteht und sich über eine Bedeutung im Klaren ist.

Ebenso verkehrt wäre es anzunehmen, Wahrheit lasse sich auf Rechtfertigung zurückführen. Aus Sicht einer normativen Pragmatik gibt es hier zwar Parallelen. ${ }^{295}$ Der Schluss von gerechtfertigten auf wahre Überzeugungen wäre aber in Analogie zu Moores ,naturalistischem Fehlschluss“ ein ungültiger. ${ }^{296}$ Auch wenn wir aufgrund konsistenter wissenschaftlicher Erkenntnisse davon ausgehen können, dass eine Überzeugung gerechtfertigt ist, legitimiert das nicht automatisch die Annahme, dass sie auch wahr ist. Die Auffassung beispielsweise, dass brennbare Materie eine Substanz namens „Phlogiston“ enthält, war

\footnotetext{
${ }^{294}$ Zur Unterscheidung semantischer und empirischer Korrektheit vgl. erneut Kap. 3.4. ${ }^{295}$ Vgl. erneut Kap. 4.6 u. 5.5. Zu der Behauptung, dass Wahrheit und Rechtfertigung nicht nur in normativer, sondern auch in logischer Hinsicht koextensional sind, vgl. Wright (2001), insbes. Kap. 1 u. 2.

296 Diese epistemologische Lesart des „,naturalistischen Fehlschlusses“ geht auf H. Putnam zurück. Vgl. Putnam (1978), 108 f. u. Rorty (1982), XXV.
} 
während des 17. und 18. Jahrhunderts eine gerechtfertigte Überzeugung. Wie wir heute aber wissen, war sie zugleich falsch. Die Frage, ob eine gerechtfertigte Überzeugung tatsächlich auch wahr ist, lässt sich sinnvollerweise immer stellen. Insofern nicht auszuschließen ist, dass eine gegebene Überzeugung gerechtfertigt und trotzdem falsch ist, sollte Wahrheit und Rechtfertigung voneinander unterschieden werden.

\subsection{Konsistenzproblem}

Auf den Unterschied zwischen Wahrheit und Rechtfertigung geht ein gewichtiger Einwand zurück, der gegenüber der Kohärenztheorie wiederholt vorgebracht wurde. Er richtet sich vor allem gegen das Kriterium der Konsistenz, die Mindestanforderung für ein kohärentes System von Überzeugungen. Ich werde daher vereinfachend vom Konsistenzproblem sprechen. Demnach kann es verschiedene in sich konsistente Systeme von Überzeugungen geben, die untereinander allerdings widersprüchlich sind. ${ }^{297}$ Ein drastisches Beispiel hierfür wäre ein Märchen, das in sich vollkommen konsistent und schlüssig ist. ${ }^{298}$ Wenn Kohärenz im Sinne von Konsistenz das entscheidende Kriterium für Wahrheit sein soll, dann müssten die Sätze dieses Märchens als ebenso wahr gelten, wie die eines historischen Berichts oder einer wissenschaftlichen Studie. Dies kann aber niemand behaupten wollen. Die Kohärenztheorie erweckt daher den Eindruck, „,öllig verfehlt“" zu sein.

Nun ist es sicherlich übertrieben zu behaupten, bei einem Märchen handle es sich um ein konsistentes Set an Überzeugungen. Selbst wenn die Geschichte aus einer Reihe von Sätzen besteht, die widerspruchsfrei zusammenhängen, handelt es sich doch nicht um Überzeugungen, die der Autor oder der Leser für wahr hält. ${ }^{299}$ Es gibt aber auch weniger drastische Beispiele, die diesen Einwand stützen. So ist durchaus vorstellbar, dass es zwei Überzeugungen gibt, die beide jeweils Teil eines konsistenten Systems von Überzeugungen sind, die aber zueinander im Widerspruch

297 Vgl. Russell (1906/07), 32 ff., Schlick (1934), 86 f., Davidson (1986), 309 ff. u. Davidson (1990), 305.

${ }^{298}$ Vgl. Schlick (1934), 86.

${ }^{299}$ Vgl. Künne (2003), $381 \mathrm{f}$. 
stehen. Ein solcher Fall findet sich beispielsweise in der Geometrie. Dort wird bekanntlich zwischen euklidischer und nichteuklidischer oder differentialer Geometrie unterschieden. Beides sind konsistente Systeme für wahr gehaltener Sätze, die sich jedoch in einem wichtigen Punkt unterscheiden. Nichteuklidische Geometrien verneinen das sogenannte Parallelenaxiom. ${ }^{300}$ Das bedeutet, dass sowohl dieser Satz als auch seine Verneinung Teil eines kohärenten Systems sind und somit als wahr gelten müssen. Die beiden Sätze zusammen können aber nicht wahr sein. Dagegen spricht das Konsistenzkriterium beziehungsweise das Gesetz vom ausgeschlossenen Widerspruch. Wir müssen also davon ausgehen, dass eines der beiden Systeme eine falsche Überzeugung enthält, obwohl es als kohärent gilt. Der Kohärenztheoretiker ist jedoch nicht in der Lage zu sagen, welches es ist. Keine der erwähnten Zusatzanforderungen (Reichhaltigkeit, Zusammenhang, Einfachheit) stellt eine überzeugende Entscheidungshilfe dar.

Er könnte allerdings entgegnen, dass die Messlatte hier unnötig hoch gelegt wird. Schließlich hat niemand ausschließlich wahre Überzeugungen. Es wäre also unsinnig zu erwarten, ein kohärentes Überzeugungssystem müsse vollkommen konsistent sein und dürfe keinerlei falsche Überzeugungen enthalten. Vielmehr müsse Kohärenz als eine graduelle Angelegenheit betrachtet werden. Für die kohärenztheoretische Erklärung von Wahrheit genüge die Forderung nach größtmöglicher Konsistenz. Dass die Wahrheit der einzelnen Überzeugungen dadurch ebenfalls zu einer graduellen Angelegenheit wird, sei damit nicht behauptet. ${ }^{301}$

Eine derartige Erklärung hat soviel für sich, wie sie gegen sich hat. Sie leuchtet zwar ein. Der Einwand, dass das Kriterium der Konsistenz Widersprüchlichkeit nicht ausschließt, wird damit aber nicht entkräftet. Vielmehr legitimiert sie das Auftreten widersprüchlicher Überzeugungen

\footnotetext{
${ }^{300}$ Vgl. Thaer (2005), 3: „Und daß, wenn eine gerade Linie beim Schnitt mit zwei geraden Linien bewirkt, daß innen auf derselben Seite entstehende Winkel zusammen kleiner als zwei Rechte werden, dann die zwei geraden Linien bei Verlängerung ins unendliche sich treffen auf der Seite, auf der die Winkel liegen, die zusammen kleiner als zwei Rechte sind.“

${ }^{301} \mathrm{Zu}$ dem Vorschlag, Wahrheit im kohärenztheoretischen Sinne als die Zughörigkeit zu einem „maximal kohärenten Set von Überzeugungen“ zu verstehen, vgl. Künne (2003), 385 .
} 
in vermeintlich konsistenten Systemen. Damit aber wird die Minimalbedingung für Kohärenz wieder aufgehoben. Angenommen es lässt sich nicht ausschließen, dass ein kohärentes System von Überzeugungen Irrtümer enthält. Woher weiß ich dann, dass es sich nicht gerade bei der einen Überzeugung, um deren Wahrheit es mir geht, um einen Irrtum handelt? Dass sie sich in ein kohärentes System eingliedern lässt, ist hier offensichtlich gerade kein Beweis. Sind aber erst einmal Irrtümer zugelassen, so bietet sich dem Skeptiker wieder die Gelegenheit, seine notorischen Fragen zu stellen.

Der Kohärenztheoretiker hält es für möglich, dass ein Überzeugungssystem sowohl kohärent als auch fehlerhaft ist. Ihm steht allerdings kein Kriterium zur Verfügung, die Menge an Irrtümern zu bestimmen oder zu limitiert. Er kann also auch nicht ausschließen, dass wir uns in beträchtlichem Umfang irren. Sobald er also bei der Bestimmung von Wahrheit Irrtümer zulässt, ist seine Konzeption dem Zweifel ausgesetzt. Schließlich können Überzeugungen hinsichtlich der Wirklichkeit kohärent und trotzdem falsch sein. ${ }^{302}$ Der Kohärenztheoretiker kann sich auf keines der genannten Kriterien berufen. Er kann auch nicht einfach darauf verweisen, dass ein kohärentes System von Überzeugungen auf dem sicheren Fundament der Erfahrung ruht. Denn, wie die Diskussion der Korrespondenztheorie gezeigt hat, führt gerade die Privilegierung dieser Klasse von Überzeugungen zu skeptizistischen Einwänden.

Somit ist die kohärenztheoretische Konzeption von Wahrheit nicht die bessere Alternative zur Korrespondenztheorie, als die sie ursprünglich angetreten ist. Das Versprechen, gegenüber skeptischen Einwänden immun zu sein, kann sie nicht einlösen. Die Schwachstelle ist das Kriterium der Konsistenz. Der Einwand, dass es mehrere in sich konsistente, aber untereinander inkonsistente Überzeugungssysteme geben kann, lässt sich nicht entkräften. Dadurch wird Kohärenz zu einem unbrauchbaren Wahrheitskriterium, mag auch auf den ersten Blick einiges an dieser Theorie plausibel erscheinen. Letztlich ist Kohärenz kein geeignetes

${ }^{302} \mathrm{Zu}$ der Auffassung, dass Überzeugungen „intrinsisch wahrheitsgetreu“ sind und globale Skepsis daher unsinnig ist, vgl. Davidson (1986), 314 ff. u. Davidson (2001), 155 . 
Kriterium für die Unterscheidung von dem, was wir glauben oder für wahr halten und dem, was tatsächlich wahr ist.

\subsection{Klassischer Pragmatismus}

Die Kohärenztheorie ist nicht die einzig denkbare Reaktion auf die Korrespondenztheorie. Eine andere Alternative bietet der klassische Pragmatismus. ${ }^{303} \mathrm{Im}$ Kern vertreten Autoren wie William James oder Ferdinand C. S. Schiller die Auffassung, dass wahre Überzeugungen für denjenigen, der sie hat, einen bestimmten Nutzen haben:

„The true, " to put it very briefly, is only the expedient in the way of our thinking (...). Expedient in almost any fashion; and expedient in the long run and on the whole of course. ${ }^{304}$

Die Aufmerksamkeit gilt dabei dem jeweiligen Zweck einer Überzeugung. Wahrnehmungsüberzeugungen beispielsweise lassen sich dahingehen sortieren, ob sie uns beim Umgang mit der Wirklichkeit behilflich sind oder nicht. Theoretische Überzeugungen lassen sich hinsichtlich der Frage beurteilen, ob sie sich mit dem bisherigen Wissen und neu auftretenden Tatsachen vereinbaren lassen. Und bei metaphysischen Überzeugungen, die beispielsweise die Existenz Gottes betreffen, kann man prüfen, ob sie der emotionalen Zufriedenheit förderlich sind. Wahre Überzeugungen machen sich also immer auf eine bestimmte Weise bezahlt. Sie sind für denjenigen, der sie hat, wünschenswert, nützlich und zufriedenstellend.

Charles S. Peirce setzt den Akzent hier etwas anders. Für ihn bestimmt sich Wahrheit weniger aus dem Nutzen einer Überzeugung, als aus dem Konsens, den letztlich alle am Thema beteiligten Forscher nach einer möglicherweise länger andauernden Untersuchung erreichen. Wahrheit bedeutet also das Ende der Forschung. ${ }^{305}$ Dies lässt sich aber leicht mit der Auffassung James' vereinbaren. Der praktische Nutzen ist in Peirce' Ansatz insofern enthalten, als eine wahre Überzeugung eine solche

${ }^{303}$ Vgl. z. B. James (1991) o. Dewey (1958).

${ }^{304}$ James (1991), 98. Vgl. auch Schiller (1907), 152. Zu einer jüngeren Variante dieses Ansatzes vgl. auch Rorty (1988), 14.

${ }^{305}$ Vgl. Peirce (1878), 300. Zu einer jüngere Variante dieses Ansatzes vgl. auch Putnam (1981), 49 ff. u. Habermas (1984), 136 f. 
ist, die sich auch nach eingehender Prüfung noch bezahlt macht. Auch die Nähe zum kohärenztheoretischen Verständnis von Wahrheit ist nicht zu übersehen. Denn Wahrheit greift in der Lesart Peirce' ebenfalls auf Rechtfertigungszusammenhänge zurück. Die experimentelle, wissenschaftliche Verifikation einer Überzeugung ist ja nichts anderes als deren Rechtfertigung. Das Ziel der Forschung sind Evidenzen, die eine Hypothese empirisch oder theoretisch bestätigen. Die Beweisführung vollzieht sich dabei grundsätzlich entlang inferentiell gegliederter Argumentationen. Am Ende der Forschung stehen schließlich wahre Überzeugungen, die Teil eines konsistenten Systems sind.

\subsection{Relativitätsproblem und Problem des unbestimmten Endes}

Auch gegen die pragmatistische Wahrheitstheorie richten sich einige gewichtige Einwände. Da sind zunächst die Schwierigkeiten, die insbesondere die Peircesche Variante von der Kohärenztheorie erbt. Zumindest indirekt steht sie vor der Aufgabe, mehrere konsistente Überzeugungssysteme ausschließen oder erklären zu müssen und dabei skeptizistische Einwände zu unterbinden. Die eigentliche Kritik setzt jedoch üblicherweise woanders an. Nehmen wir zunächst die Auffassung, Wahrheit bestimme sich aus dem Nutzen einer Überzeugung. Nach einigen Überlegungen stellt sich die Frage, für wen eine gegebene Überzeugung eigentlich nützlich sein soll. ${ }^{306}$ Damit tritt zutage, was sich als das Relativitätsproblem zusammenfassen lässt.

Der Nutzen einer Überzeugung hängt von den Wünschen und Erwartungen desjenigen ab, der sie hat. Dies aber ist von Person zu Person sehr unterschiedlich. Folglich sind auch nicht für alle dieselben Überzeugungen nützlich, sondern variieren bisweilen stark. Der eine beispielsweise zieht einen Nutzen aus der Überzeugung, dass der Zug eine Verspätung eingefahren hat: Es gibt ihm mehr Zeit für das Gespräch mit der interessanten Reisebegleitung. Für den anderen dagegen ist dies überhaupt nicht nützlich: Er verpasst seinen Anschlusszug. Dieselbe Überzeugung kann also zugleich nützlich und nutzlos sein. Sie kann aber nicht zugleich wahr und unwahr sein. Der Pragmatist müsste seine Theorie

${ }^{306}$ Vgl. David (2004), $351 \mathrm{f}$. 
daher dahingehend erweitern, dass Wahrheit von Person zu Person variiert. „Wahr“ würde dann immer bedeuten „wahr für $S^{\text {“ }} .{ }^{307}$ Ein derart relativistisches Verständnis von Wahrheit würde aber sicher auf wenig Akzeptanz stoßen bei denen, die ernsthaft an einer Begriffsbestimmung interessiert sind. Wahrheit gilt schließlich als zeitlos und nicht gradierbar. $^{308}$

Dies ist auch die Grundlage eines weiteren Einwandes. ${ }^{309}$ Danach kann Nützlichkeit weder eine notwendige noch eine hinreichende Bedingung für wahre Überzeugungen sein. Denn dazu gibt es zu viele Ausnahmen. Zum einen gibt es Überzeugungen, die nützlich sind, unabhängig davon, ob sie wahr oder falsch sind. Das gilt beispielsweise für die Überzeugung, dass es außer einem selbst andere Menschen gibt. Die Wahrheit dieser Überzeugung ist noch für den größten Misanthropen nützlich. Selbst für ihn ist es von Nutzen, dass das, was er hasst, existiert. Allerdings ist die Nützlichkeit dieser Überzeugung überhaupt nicht darauf angewiesen, dass sie auch wahr ist. Auch wenn es keine anderen Menschen gibt, kann es nützlich sein, dies zu glauben. Für ein einsames Gehirn im Tank kann es nützlich sein zu glauben, es gebe andere Menschen. So wie es nützlich sein kann zu glauben, dass Gott existiert, selbst wenn dies nicht der Fall ist. ${ }^{310}$

Zum anderen gibt es wahre Überzeugungen, die sowohl nützlich als auch nutzlos oder gar irrelevant sein können. ${ }^{311}$ Für einen großen Teil unserer Überzeugungen gilt, dass sie in manchen Situationen nützlich sind, in manchen aber auch nicht. Für die allgemeine Risikoeinschätzung etwa ist die wahre Überzeugung, dass Flugzeugabstürze in der Regel für alle Insassen tödlich sind, nützlich. Für eine Person mit großer Flugangst, die unbedingt einen Flug antreten muss, hat diese Überzeugung dagegen keinen Nutzen. Für sie stellt sie vielmehr ein unnützes Hindernis dar.

307, ,S“ steht für die jeweilige Person, die die wahre Überzeugung hat.

308 Diese Kritik lässt sich auf Platons Widerlegung von Protagoras' relativistischem Wahrheitsverständnis im Theaitetos zurückführen. Vgl. Platon (1994d), 152a u. 161b 162b. Vgl. auch Putnam (1981), 55 u. 120 ff.

309 Vgl. Moore (1907/08) u. Russell (1910), 127 ff. Zum folgenden Beispiel vgl. Russell (1910), $139 \mathrm{f}$.

${ }^{310}$ Vgl. James (1991), 38 u. 131.

${ }^{311}$ Vgl. Moore (1907/08), 43 ff. 
Andere Überzeugungen, wie etwa die, dass zwei und zwei vier ergeben oder dass in Berlin jede Woche mehrere tausend Bierflaschen zu Bruch gehen, sind zwar durchaus wahr. Zugleich sind sie aber auch in den meisten Lebenssituationen irrelevant. ${ }^{312}$ Und schließlich ist es auch nicht schwer sich vorzustellen, dass falsche Überzeugungen ebenso nützlich sein können wie wahre. Irre ich mich beispielsweise hinsichtlich der Uhrzeit oder eines Ortes und entgehe dadurch einem Unglück, so hat dies für mich einen beträchtlichen Nutzen.

Es stellt sich also heraus, dass die Nützlichkeit einer Überzeugung nicht nur davon abhängt, wer sie hat. Sie ist auch relativ zu der Situation, in der sie auftaucht. Der Ausdruck ,wahr“ muss nun nicht nur als ,wahr

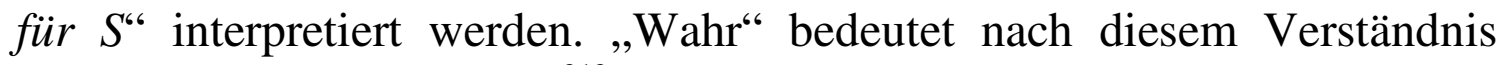
vielmehr ,wahr für $S$ in $U^{“} .{ }^{313}$ Eine solche Auskunft ist für das Verständnis von Wahrheit jedoch wertlos. Schließlich soll eine wahre Überzeugung unabhängig von Ort und Zeitpunkt wahr sein. Zudem zeigen die Beispiele, dass Wahrheit und Nutzen in vielen Fällen vollkommen unabhängig voneinander sind. Wenn sich hier auch Gemeinsamkeiten beobachten lassen, so dürfen die Begriffe dennoch nicht gleichgesetzt werden. Es mag sein, dass wir in sehr vielen oder sogar den meisten Fällen von wahren Überzeugungen profitieren. In nicht wenigen Fällen ist Irrtum allerdings ebenso nützlich, wie Wahrheit hinderlich ist. Ein Kriterium aber, das solche Ausnahmen zulässt, ist ebenso wenig zu gebrauchen, wie der erwähnte Relativismus. Die Orientierung an der praktischen Relevanz kann nicht verhindern, dass der Pragmatismus an dem Versuch einer Bestimmung von Wahrheit scheitert.

Das gilt auch für die Auffassung, wonach Wahrheit das Ende der Forschung ist. Das Kriterium ist hier zugleich das Problem. Man kann es als Problem des unbestimmten Endes bezeichnen. Es stellt sich nämlich die Frage, wie man sich ein Ende der Forschung überhaupt vorzustellen hat. Es ist in verschiedener Hinsicht unklar, ob es das überhaupt geben kann. Die Wahrheit einer Überzeugung kann sicher nicht von der Zustimmung eines letzten, überlebenden Individuums abhängen. ${ }^{314}$ Denn dann gäbe es

${ }^{312}$ Vgl. James (1991), 102.

${ }^{313}, U^{“}$ steht hier für die jeweiligen Umstände, in denen die wahre Überzeugung vorkommt.

${ }^{314}$ Vgl. Russell (1989), 145. 
solange keine Wahrheit, bis der letzte Forscher im letzten Moment seines Lebens ein letztes Urteil abgegeben hat. Das Ende der Forschung kann daher nur eine ideale Grenze sein, die nicht tatsächlich erreicht wird, sondern der sich die verschiedenen Untersuchungen annähern. ${ }^{315}$ Allerdings ist diese Interpretation nicht weniger problematisch. Denn auch als Ideal ist das Ende der Forschung ein zweifelhaftes Wahrheitskriterium. Aus mindestens zwei Gründen ist fraglich, ob überhaupt eine Annäherung an diese Grenze möglich ist. ${ }^{316}$

Der eine ist, dass eine solche Annäherung nur als ein Moment vorstellbar ist, in dem alle relevanten Informationen für die Beurteilung einer bestimmten Überzeugung zur Verfügung stehen. Es ist aber schwer einzusehen, dass es einen solchen Moment geben kann. Denn was es heißt, dass eine Überzeugung durch alle relevanten Informationen gestützt ist, lässt sich gar nicht genau sagen. Die Menge der Informationen, die eine bestimmte Überzeugung stützt, ist auf vielfältige Weise im System der Überzeugungen der beteiligten Forscher verzweigt. Für jede Überzeugung gibt es eine Fülle von Hintergrundüberzeugungen, die Einfluss auf den Kontext der epistemische Situation haben. Dies ist eine Konsequenz des Überzeugungsholismus, der jeglicher Forschung zugrunde liegt. In einer geeigneten Gesamtsituation kann daher jede Information für jede beliebige Überzeugung relevant sein. Um also tatsächlich über alle, für eine bestimmte Überzeugung relevanten Informationen zu verfügen, muss man schlicht über alle Informationen verfügen. So verstanden ist zwar ein Ende der Forschung als eine Art universelles Weltwissen vorstellbar. Der Grad einer sich steigernden Annäherung an dieses Ende bleibt jedoch prinzipiell unbestimmbar.

Der andere Grund ist der folgende. Selbst wenn man annimmt, jemand wäre in der Lage, ein Maximum an Informationen hinsichtlich einer bestimmten Überzeugung zusammenzutragen, so deutet nichts darauf hin, dass er einen solchen Zustand auch erkennen könnte. Wie sollte er sicher sein, dass er sich mit seiner Theorie dem Ende der Forschung maximal angenähert hat? Er könnte ebenso gut aus Erschöpfung oder mangels

\footnotetext{
${ }^{315}$ Vgl. Putnam (1981), 55 f.

${ }^{316}$ Zum Folgenden vgl. Wright (2001), 64 ff.
} 
weiterer Ideen aufgehört haben $\mathrm{zu}$ forschen. ${ }^{317}$ Die wissenschaftliche Forschung macht ohne Zweifel Fortschritte. Diese erkennen wir aber erst im Rückblick, wenn wir eine Verbesserung gegenüber vorausgegangenen Theorien sehen. Um zu verstehen, was wissenschaftlicher Fortschritt ist, benötigen wir die Idee einer asymptotischen Annäherung an eine ideale Grenze jedenfalls nicht. Ebenso wenig können wir mit ihrer Hilfe erklären, wie es immer wieder $\mathrm{zu}$ unvorhergesehenen Revolutionen in der Wissenschaft kommt. Das Ende der Forschung ist damit als Test für Wahrheit doppelt ungeeignet. Weder als konkretes noch als ideales Ende ist es erreichbar. Im einen Fall stünde Wahrheit nur dem letzten Menschen auf Erden zur Verfügung. Im anderen Fall wäre es ein unerreichbares und unerkennbares Ideal.

Der bisherige Überblick erlaubt das folgende Zwischenresümee. Die Korrespondenztheorie trägt der realistischen Intuition Rechnung, dass es eine objektive Wirklichkeit gibt, auf die sich unsere Überzeugungen und Aussagen beziehen. Sie scheitert aber daran, dass sich die Übereinstimmung der Überzeugungen oder Aussagen mit der Wirklichkeit nicht annehmen lässt, ohne dass dabei skeptische Fragen aufgeworfen werden. Die Kohärenztheorie verbleibt innerhalb von Sprache und Überzeugungen und kann dadurch dem Außenweltskeptizismus ausweichen. Allerdings stellen sich ihr die Probleme, mehrere untereinander inkonsistente Überzeugungssysteme und einen globalen Irrtum nicht ausschließen zu können. Die Wahrheitstheorien des klassischen Pragmatismus sind dem methodischen Minimalismus verpflichtet, wonach philosophisch irrelevant ist, was in praktischer Hinsicht keinen Unterschied macht. Allerdings sind die Kriterien, die sie anbieten, letzten Endes unbrauchbar. Wahrheit und Nutzen fallen nicht in allen Fällen zusammen. Und das Ende der Forschung lässt sich weder bestimmen noch erkennen.

\subsection{Deflationismus}

Die Probleme der klassischen Wahrheitstheorien geben Grund zur Annahme, dass es so etwas wie ein Wesen der Wahrheit nicht gibt. Eine Definition „des Wahren“ oder ,der Wahrheit“ scheint nicht möglich.

${ }^{317}$ Vgl. Rorty (1986), 338. 
Wahrheit verhält sich offenbar nicht wie ein gewöhnlicher Teil der Wirklichkeit, dessen allgemeine Merkmale sich erforschen und beschreiben lassen. Die grammatische Oberfläche erweckt zwar den Anschein, mit dem Wort „wahr“" werden Überzeugungen spezifische Eigenschaften zugeschrieben, deren allgemeine Struktur sich durch die geeignete philosophische Theorie bestimmen lässt. Der Ausdruck ,ist wahr" verhält sich jedoch nicht wie ein herkömmliches Prädikat wie beispielsweise ,ist magnetisch“ oder ,ist diabetisch“. ${ }^{318}$ Diese beziehen sich auf Phänomene oder Eigenschaften, die sich in den entsprechenden Wissenschaften als Magnetismus oder Diabetes mehr oder weniger exakt bestimmen lassen. Auf das Wahrheitsprädikat lässt sich das jedoch nicht ohne weiteres übertragen. Wahrheit als solche scheint weder der wissenschaftlichen noch der philosophischen Analyse zugänglich. Wir sollten also nicht fragen, was Wahrheit ist, sondern was wir tun, wenn wir von den entsprechenden Redewendungen Gebrauch machen. Dies erscheint mir als die zwingende Konsequenz aus dem Scheitern der zuvor erörterten Wahrheitstheorien.

Damit komme ich auf die eingangs formulierte Aufgabe zurück, Funktion und Bedeutung von Wahrheit mit den Mitteln des bisher entwickelten normativ-inferentiellen Theoriemodells weiter zu präzisieren. Der Vorschlag war zunächst, den Gebrauch von ,wahr“ als die Übernahme oder das Sich-zu-eigen-machen einer Festlegung zu verstehen. Wer eine Überzeugung oder Behauptung als wahr bezeichnet, legt sich selbst auf sie fest. Er bestätigt den Anspruch, der mit der ursprünglichen Festlegung erhoben wird: Das Geglaubte soll wahr sein, denn Glauben heißt Fürwahrhalten. Man kann also sagen, dass mit der Verwendung des Wahrheitsprädikates eine Art Zustimmung, Wertschätzung oder auch Empfehlung einhergeht. ${ }^{319}$ Dies ist offensichtlich in Fällen, in denen jemand in Reaktion auf eine bestimmte Behauptung sagt: „Das ist wahr.“ oder „Das stimmt.“ Es trifft aber auch dort zu, wo jemand eine Behauptung direkt aufgreift und beispielsweise sagt: „Es ist wahr, dass Paris die Hauptstadt von Frankreich ist.“

Etwas als wahr zu bezeichnen, bedeutet damit zunächst einmal, ihm gegenüber eine wertende Haltung einzunehmen. In der zuvor eingeführten

\footnotetext{
${ }^{318} \mathrm{Vgl}$. Horwich (1998b), 2.

${ }^{319}$ Vgl. Rorty (1986), 334, Rorty (1988), 16 o. Brandom (1994), 287.
} 
Theoriesprache ausgedrückt kommt dies dem Einnehmen einer normativen Einstellung, sowie der Übernahme eines normativen Status gleich. ${ }^{320}$ Durch das Wahrheitsprädikat wird eine Behauptung zustimmend oder empfehlend bewertet. Sie wird als wahr betrachtet und behandelt. Diese normative Einstellung schlägt sich darin nieder, dass jemand den Status der Festlegung für sich übernimmt. Mit dieser Übernahme der ursprünglichen Behauptung geht zugleich aber die Zuschreibung des Status der Berechtigung einher. Wer eine Behauptung als wahr betrachtet und behandelt, erteilt dazu rückwirkend die Erlaubnis. Vereinfacht ausgedrückt bringt ein Diskursteilnehmer also mit dem Ausdruck „wahr“ die Einschätzung zum Ausdruck, dass eine Behauptung zumindest für den Moment hinlänglich gerechtfertigt ist. ${ }^{321}$

Der Fokus dieser Erklärung liegt ganz auf den performativen und normativen Aspekten von Wahrheitszuschreibungen. Der Gegenstand der jeweiligen Überzeugungen oder Behauptungen wird dabei nicht eigens thematisiert. Etwas als wahr zu bezeichnen, bedeutet demnach auch nicht, es in Bezug auf seinen Gehalt zu beschreiben. Ob mit der Zertifizierung durch den Ausdruck „wahr“ eine unabhängige Eigenschaft wie beispielsweise die Übereinstimmung mit der Wirklichkeit oder ein spezifischer Nutzen zum Ausdruck kommt, ist nicht von Interesse. Die These ist vielmehr, dass man mit „Es ist wahr, dass Paris die Hauptstadt von Frankreich ist.“ prinzipiell dasselbe behauptet wie mit „Paris ist die Hauptstadt von Frankreich.“ und umgekehrt. Die Bedeutung der Aussage ändert sich nicht, wenn sie durch den Zusatz „Es ist wahr, dass ...“ Zustimmung oder Wertschätzung erfährt.

Somit ergibt sich mit diesem Erklärungsansatz ein deflationistisches Bild von Wahrheit. ${ }^{322}$ Es zielt darauf ab, dem alten metaphysischen Rätsel vom Wesen der Wahrheit einen Großteil seiner Brisanz zu nehmen, indem dem Begriff jede philosophisch tragende Rolle abgesprochen wird. Schließlich sieht es so aus, als würde mit dem Zusatz, dass eine Überzeugung oder Behauptung wahr ist, überhaupt nichts Substantielles gesagt. Es hat vielmehr den Anschein, als wäre das Wahrheitsprädikat

${ }^{320}$ Vgl. hierzu erneut Kap. 4.9 f. u. Brandom (1994), 288 f.

${ }^{321}$ Vgl. Rorty (1988), $16 \mathrm{f}$.

${ }^{322} \mathrm{Zu}$ einem Überblick über einige Autoren, die einen vergleichbaren Ansatz vertreten, vgl. Horwich (1998b), 5 Anm. 2. 
nicht mehr als rhetorischer Zierrat. Außer der ausdrücklichen Zustimmung oder Unterstützung scheint ihm keine weitere Funktion zuzukommen. Und selbst dabei ist noch zweifelhaft, ob nur das Wahrheitsprädikat sie erfüllen kann. Wie ich noch zeigen werde, gibt es andere Redensarten, die dafür ebenso gut geeignet sind. Daher ist die Frage erlaubt, ob es sich bei Wahrheit überhaupt um ein echtes Prädikat handelt, ob also der Ausdruck „,ist wahr“ nicht nur der Form, sondern auch der Substanz nach ein Prädikat ist.

Vertreter der Redundanztheorie der Wahrheit bestreiten dies. ${ }^{323}$ Sie bezweifeln, dass der Ausdruck ,ist wahr“ dazu dient, Dingen eine Eigenschaft zuzuschreiben. Die letzten Abschnitte können diese Einschätzung nur bestätigen. Tatsächlich gibt es sprachliche Zusammenhänge, in denen der Ausdruck ,ist wahr" keinerlei explanatorischen oder semantischen Mehrwert erzeugt. Damit sind all die Fälle gemeint, in denen das, was als wahr bezeichnet wird, direkt wiedergegeben wird. Die Behauptung „Es ist wahr, dass Paris die Hauptstadt von Frankreich ist.“ ist dafür ein Beispiel. Was hier als wahr bezeichnet wird, ist selbst ausdrücklich Teil der Äußerung. In Fällen wie diesen erscheint die Wahrheitszuschreibung trivial. Wahrheit ist nur insofern eine Eigenschaft, als sie all die unterschiedlichen Sätze verbindet, in denen der Ausdruck ,ist wahr" vorkommt. Das ist zugegebenermaßen nicht sehr informativ. Man riskiert also nicht viel, wenn man eine solche Eigenschaft als überflüssig und verzichtbar bezeichnet.

Anhand der genannten Fälle gewinnt man den Eindruck, dass „Es ist wahr, dass $p$." nur eine umständliche Art und Weise ist zu sagen, dass $p$. Die Zuerkennung von Wahrheit ist hier letztlich nichts anderes als die redundante Wiederholung einer Überzeugung. Was ihren Gehalt betrifft, ist kein Unterschied erkennbar. Aber auch in normativer Hinsicht besteht hier offenbar kein grundlegender Unterschied. In beiden Fällen handelt es sich um die Festlegung auf einen Gehalt, nämlich genau denselben. Mit

${ }^{323}$ Vgl. insbes. Frege (1994), 40 ff., Ramsey (1927) u. Ayer (2001), 84 ff. P. Strawson vertritt in seiner sogenannten performativen Theorie der Wahrheit anfangs dieselbe Auffassung. Vgl. Strawson (1954), 84. Für Brandom hat ,ist wahr“ nicht einmal die grammatikalische Form eines Prädikats. Gemäß der von ihm vertretenen ProsatzTheorie der Wahrheit handelt es sich hierbei um einen „prosatzbildenden Operator“. Vgl. Brandom (1994), $301 \mathrm{ff}$. 
dem Wahrheitszusatz wird dies lediglich explizit gemacht und personalisiert. Wer eine Überzeugung oder Behauptung als wahr bezeichnet, veröffentlicht dadurch seine persönliche, normative Einstellung zu ihr. Man kann daher durchaus zu dem Schluss kommen, dass Wahrheit in diesen Zusammenhängen trotz irreführender grammatischer Oberflächenwirkung kein echtes Prädikat ist. Die Verwendung des Ausdrucks „,ist wahr“ fügt der jeweiligen Äußerung nichts hinzu, was sie nicht ohnehin schon beinhaltet. Somit scheint mit dem Äquivalenzschema

(̈̈S) Es ist wahr, dass $p$, genau dann, wenn $p$.

alles gesagt zu sein, was sich zum Wahrheitsbegriff sagen lässt. ${ }^{324}$ Der Begriff der Wahrheit erschöpft sich offensichtlich in dem, was die Substitutionsinstanzen dieses Schemas wiedergeben.

Zumindest in der großen Zahl der unkontroversen Fälle leuchtet (ÄS) intuitiv ein. ${ }^{325}$ Nichts könnte klarer sein, als die verschiedenen Instanzen, die sich aus dem Schema ableiten lassen. Wenn es aber gleichgültig ist, ob ein Satz oder eine Behauptung eine Wahrheitszuschreibung enthält oder nicht, wozu gibt es den Ausdruck ,wahr“ dann überhaupt? Immerhin ist er ein fester Bestandteil unserer Sprache. Anstatt vorschnell von Redundanz zu sprechen, halte ich es daher für ratsamer, das Schema eher als einen Hinweis auf die Relevanz des Wahrheitsbegriffs zu verstehen. Denn es lässt sich zeigen, dass es hier durchaus Unterschiede gibt, wenngleich sie nicht den Inhalt der fraglichen Sätze betreffen.

${ }^{324}$ Es gibt verschiedene Versionen dieses Schemas. A. Tarskis Variante ist Teil seiner berühmten Konvention $W: S$ ist wahr genau dann wenn $p$ (wobei „, $S$ “ die Bezeichnung eines Satzes der Objektsprache und ,,$p^{“}$ die Übersetzung dieses Satzes in der Metasprache ist). Vgl. Tarski (1935), 305 f. Quine versteht das Schema als eine Form der Zitattilgung: ,p“ ist wahr genau dann, wenn $p$. Vgl. Quine (1970), 13. P. Horwich wendet es auf Propositionen an: Die Proposition, dass $p$, ist wahr genau dann, wenn $p$. Vgl. Horwich (1998b), 10 u. 35. Ich werde mich im Folgenden mit der vereinfachten Variante begnügen, die Horwichs Vorschlag am nächsten kommt.

${ }^{325} \mathrm{Zu}$ den kontroversen Fällen vgl. Horwich (1998b), 40 ff. u. 128 u. Field (2001), 143 ff. Dazu zählen z. B. Instanzen, die den Ausdruck ,wahr“ selbst enthalten und dadurch zu Widersprüchen führen, Aussagen, die leere Namen („Pegasus“, „Einhorn“ etc.) oder vage Ausdrücke (,glatzköpfig“, „Hügel“ etc.) enthalten, die ethische Grundsätze formulieren oder die sich auf zukünftige Ereignisse beziehen. 
Da ist zum einen die bereits erwähnte Besonderheit des Sprechaktes. ${ }^{326}$ Es ist eben nicht genau das Gleiche, ob ich sage: „Paris ist die Hauptstadt von Frankreich.“ oder: „Es ist wahr, dass Paris die Hauptstadt von Frankreich ist." Im einen Fall halte ich das, was ich sage, lediglich für wahr. Im anderen mache ich es zusätzlich explizit. Das Wahrheitsprädikat gibt mir also die Möglichkeit, meine normative Einstellung gegenüber einer Behauptung offen zum Ausdruck zu bringen. Das kann nützlich sein, wenn es beispielsweise darum geht, Zweifel bei anderen Gesprächsteilnehmern auszuräumen. Ich verfüge damit über ein zusätzliches rhetorisches Mittel in der Praxis des Gebens und Verlangens von Gründen. Allerdings gibt es hier auch Alternativen. So kann ich ebenso gut sagen: „Ich glaube sehr wohl, dass ...", „Ich bin fest davon überzeugt, dass ..." etc. Das Wahrheitsprädikat ist also nicht das einzige Mittel, das mir zu diesem Zweck zur Verfügung steht.

Entscheidend ist daher auch ein anderer Unterschied. Wie das Schema (ÄS) zeigt, fügt das Wahrheitsprädikat einem Satz oder einer Behauptung inhaltlich nichts hinzu. Die beiden Teilsätze sind material äquivalent. Dass sie dies auch in logischer Hinsicht sind, ist damit allerdings nicht gesagt. Vielmehr wird ein deklarativer Satz unter Verwendung des Wahrheitsprädikates so reformuliert, dass er zu einem quantifizierbaren Gegenstand wird. Anders gesagt ist es mit Hilfe des Wahrheitsprädikates möglich, den gesamten Gehalt eines Satzes so auszudrücken, dass er den Status eines singulären Terminus erhält. Das Schema verdeutlicht dies, indem es einen Satz explizit zum Gegenstand der Wahrheitszuschreibung macht. $^{327}$ Was also eine Aussage wie „Paris ist die Hauptstadt von Frankreich.“ von Reformulierungen wie „Es ist wahr, dass Paris die Hauptstadt von Frankreich ist.“ oder „Die Proposition, dass Paris die Hauptstadt von Frankreich ist, ist wahr.“ oder ,(Der Satz) ,Paris ist die Hauptstadt von Frankreich.' ist wahr." unterscheidet, ist der Umstand, dass diese sie als quantifizierbare Einheit enthalten. In dieser Funktion ist das Wahrheitsprädikat tatsächlich ohne Alternative.

Um die Tragweite dieses Unterschiedes $\mathrm{zu}$ verstehen, ist es erforderlich, eine andere Art von Beispielen zu betrachten. Es gibt nämlich

\footnotetext{
${ }^{326}$ Vgl. auch Strawson (1954).

${ }^{327} \mathrm{Vgl}$. Horwich (1998b), 32.
} 
sprachliche Zusammenhänge, in denen die Redundanz des Wahrheitsbegriffs nicht so offensichtlich ist, wie es bisher den Anschein hatte. Gemeint sind Fälle, in denen das, was als wahr bezeichnet wird, nicht direkt wiedergegeben wird. Das, worauf sich das Wahrheitsprädikat bezieht, ist hier nur verdeckt oder indirekt gegeben. Typische Beispiele sind Generalisierungen und sogenannte blinde Zuschreibungen wie „Alles, was Hannah über Karl sagt, ist wahr.“, „Was Maria soeben behauptet hat, ist wahr." oder „Fermats letzter Satz ist wahr." Auf den ersten Blick ist nicht ersichtlich, inwiefern in Fällen wie diesen ein Unterdrücken des Wahrheitsprädikates zu äquivalenten Sätzen oder Behauptungen führt. Aus syntaktischen Gründen ist es vielmehr unentbehrlich. Schließlich handelt es sich bei „Alles, was Hannah über Karl sagt.“ ebenso wenig um einen grammatisch sinnvollen Satz, wie bei „Was Maria soeben behauptet hat.“ oder „Fermats letzter Satz.“ Es ist nicht einzusehen, wieso das Wahrheitsprädikat hier redundant sein soll.

Fälle wie diese unterscheiden sich von denen der vorausgehenden Abschnitte in einem entscheidenden Punkt. Während sich die Wahrheitszuschreibung dort direkt auf die angeführte Sätze oder Behauptungen bezog, lässt sich der Gegenstand der Zuschreibung hier nicht genau identifizieren. Dennoch findet sie statt. Und genau darin liegt nun das entscheidende und eigenständige Charakteristikum des Wahrheitsprädikates. Denn es ermöglicht uns die zustimmende, wertschätzende oder empfehlende Bezugnahme auf Sätze oder Behauptungen auch dann, wenn wir nicht in der Lage sind, sie direkt oder im Einzelnen auszudrücken. ${ }^{328}$ Durch das Wahrheitsprädikat sind wir in der Lage, unsere normative Einstellung auch dort zum Ausdruck zu bringen, wo die fraglichen Inhalte nicht unmittelbar oder in ihrer Gesamtheit vorliegen. Wir mögen nicht jede einzelne Äußerung Hannahs über Karl kennen. Dennoch können wir sie für eine unfehlbare Informationsquelle halten, etwa weil sie seine langjährige Partnerin ist. Auch was Maria soeben behauptet hat, kann uns im Einzelnen entgangen oder entfallen sein. Dennoch können wir es als wahr bezeichnen, weil wir

${ }^{328}$ Diese Einsicht geht auf Quine zurück. Vgl. Quine (1970), 11. Vgl. auch Leeds (1978), 121 f., Horwich (1998b), 2 f., Soames (1999), 230 f., Field (2001), 28 u. 119 ff. o. Williams (2001a), $147 \mathrm{f}$. 
uns beispielsweise daran erinnern, dass wir der Behauptung soeben bereits zugestimmt haben. Und von Zahlentheorie müssen wir überhaupt keine Ahnung haben, um sagen zu können, dass Fermats letzter Satz wahr ist. Wir können uns beispielsweise auf die allgemein bekannte Expertise anderer berufen.

Mit Blick auf sprachliche Zusammenhänge dieses zweiten Typs muss Wahrheit daher sehr wohl als ein echtes und gehaltvolles Prädikat gelten. Allerdings dient es nicht der Zuschreibung einer Eigenschaft, die sich durch eine eigene, reduktionistische Theorie abschließend erklären lässt. So gesehen wäre es irreführend, von einer allgemeinen Natur oder Substanz zu reden, die den verschiedenen Redewendungen, in denen der Ausdruck „wahr“ vorkommt, zugrunde liegt. Wahrheit ist kein explanatorisch bedeutsamer Grundbegriff. Er eignet sich nicht zur Erklärung konzeptioneller oder metaphysischer Zusammenhänge. Richtig ist vielmehr, dass er in seinen unterschiedlichen Verwendungsweisen eine expressive Funktion erfüllt. Er dient dazu, logische Eigenheiten und Zusammenhänge zum Ausdruck zu bringen. Nur in diesem Sinne ist die Rede von einer gemeinsamen Eigenschaft gerechtfertigt. Und in diesem Sinne ist Wahrheit auch nicht redundant. Es ist daher angemessen, sie als logische Eigenschaft oder logischen Begriff $\mathrm{zu}$ bezeichnen. ${ }^{329}$ Der entscheidende Sinn und Zweck des Wahrheitsprädikates besteht darin, Generalisierungen und indirekte Behauptungen $\mathrm{zu}$ ermöglichen. Kein anderes sprachliches oder logisches Mittel verfügt über diese Ausdruckskraft auf vergleichbar einfache und intuitive Weise. Darum gibt es das Wahrheitsprädikat, und darum ist es von unschätzbarem Wert. In den folgenden Abschnitten werde ich diesen Gedanken weiter vertiefen und untersuchen wie es kommt, dass es hier zum Wahrheitsprädikat tatsächlich keine Alternative gibt. Zu diesem Zweck werde ich die sprachlich-logischen Hintergründe der Funktions- und Verwendungsweisen dieses Prädikates detailliert beleuchten.

${ }^{329}$ Vgl. Horwich (1998b), 2 u. 37 f., Künne (2003), 91 u. 338 o. Williams (2004), 121. 


\subsection{Wahrheit und Quantifikation}

Das Wahrheitsprädikat ermöglicht indirekte Behauptungen, deren Gehalt und Ursprung uns verschlossen sind. Zudem versetzt es uns in die Lage, eine Vielzahl von Behauptungen mit einem einzigen, endlichen Satz $\mathrm{zu}$ erfassen. Vergleichbare Effekte erzielen wir nur mit großem theoretischem Aufwand. Eine partikulare Aussage wie „Was Maria soeben behauptet hat, ist wahr." ließe sich nur durch eine unendliche Disjunktion ausdrücken, wie etwa:

(24) Wenn Maria soeben behauptet hat, dass Schnee weiß ist, dann ist Schnee weiß, oder wenn Maria soeben behauptet hat, dass Gras grün ist, dann ist Gras grün, oder wenn Maria soeben behauptet hat, dass Vögel fliegen können, dann können Vögel fliegen ... usw.

Um eine generelle Aussage wie „Alles, was Hannah über Karl sagt, ist wahr." auf eine andere Weise auszudrücken, müssten wir eine unendlich lange Konjunktion der folgenden Art bilden:

(25) Wenn Hannah über Karl sagt, dass er Tiramisu liebt, dann liebt er Tiramisu, und wenn Hannah über Karl sagt, dass er schnarcht, dann schnarcht er, und wenn Hannah über Karl sagt, dass er täglich joggen geht, dann geht er täglich joggen ... usw.

Dies sind aber schon allein deshalb schlechte Alternativen, weil uns für die Formulierung solcher Disjunktionen oder Konjunktion die Ressourcen fehlen. Niemand könnte sie äußern oder aufschreiben. Daher ist der Versuch, Generalisierungen oder indirekte Behauptungen auf diese Weise explizit zu machen, zum Scheitern verurteilt. Der Ausdruck „,... usw.“ ist hier nur ein Notbehelf, dem allerdings nicht der Status und die Funktion eines gültigen generalisierenden Ausdrucks zukommt.

Die übliche Vorgehensweise bei der Darstellung von unendlichen Disjunktionen und Konjunktionen ist daher auch eine andere. Das adäquate Mittel der Wahl ist hier die Quantifikation. ${ }^{330}$ Dabei lassen sich zwei

${ }^{330}$ Im Folgenden werde ich mich auf Konjunktionen beschränken. Was für sie gilt, lässt sich auf Disjunktionen übertragen. 
Schritte unterscheiden. Zunächst ersetzt man den variierenden Teil der einzelnen Aussagen durch Variablen. Dadurch ergibt sich ein Schema wie:

(26) Wenn Hannah über Karl sagt, dass $p$, dann $p$.

Dieses Schema lässt sich dann durch eine universelle Quantifikation verallgemeinern. Das sieht in etwa folgendermaßen aus:

(27) Für alle $p$, wenn Hannah über Karl sagt, dass $p$, dann $p$.

So lassen sich sämtliche Aussagen der Konjunktion in (25) in einer einzigen zusammenfassen. Der Vorteil dieser Vorgehensweise ist, dass sie auf fragliche Mittel wie den Ausdruck „... usw.“ verzichtet und lediglich Standardinstrumente der Logik verwendet.

Wie es scheint erhalten wir mit (27) eine Generalisierung der unendlichen Konjunktion, die zudem ohne das Wahrheitsprädikat auskommt. Allerdings entspricht das Ergebnis, so wie es ist, nicht den üblichen Konventionen der objektualen Quantifikation. ${ }^{331}$ Für gewöhnlich geben universelle Quantifikationen Auskunft darüber, dass alle Gegenstände eines bestimmten Bereichs eine bestimmte Eigenschaft haben. Existentielle Quantifikationen informieren entsprechend über die Existenz mindestens eines solchen Gegenstandes. Buchstaben wie „, $p^{\text {“ }}$ dienen als Variablen, die mit diesem Gegenstandsbereich assoziiert werden. Sie markieren als Platzhalter eine Position, die ein beliebiges Vorkommnis dieses Bereichs einnehmen kann. Syntaktisch werden diese Variablen als singuläre Termini angesehen.

Wie sich aber herausstellt, kann das letzte Vorkommen von , $p$ “ in dem generalisierten Schema (27) nicht auf diese Weise interpretiert werden. Sollen seine Instanzen grammatisch wohlgeformte Sätze sein, so kann , ,p“ hier nicht die Position eines Gegenstandes einnehmen. Denn das Resultat wäre Nonsens, ganz gleich, ob die Gegenstände, über die quantifiziert wird, Propositionen oder Sätze sind. Im einen Fall ergeben sich Aussagen wie:

${ }^{331}$ Vgl. z. B. Soames (1997), 6 f., Horwich (1998b), 31 f. o. Künne (2003), 356 f. 
(28) Wenn Hannah über Karl die Proposition äußert, dass er Tiramisu liebt, dann die Proposition, dass er Tiramisu liebt.

Im anderen ergeben sich solche der folgenden Art:

(29) Wenn Hannah über Karl den Satz äußert: „Er liebt Tiramisu.“, dann der Satz: „Er liebt Tiramisu.“

Beide sind offensichtlich unvollständig. ${ }^{332} \mathrm{Um}$ sie $\mathrm{zu}$ vervollständigen, müssten wir das letzte Vorkommen der Variable , $p “$ in (27) durch ein Prädikat ergänzen. Dadurch würde in der jeweiligen Instantiierung auch nach dem Partikel „dann“ ein vollständiger Satz stehen. Naheliegend ist hier natürlich die Ergänzung durch das Prädikat „ist wahr“ oder eine Paraphrase desselben:

(30) Für alle $p$, wenn Hannah über Karl sagt, dass $p$, dann ist $p$ wahr.

Für eine solche Ergänzung müsste es aber eine plausible Erklärung geben, soll sie nicht vollkommen willkürlich erscheinen. Ist unser Ehrgeiz zudem der, das Wahrheitsprädikat durch eine derartige Quantifikation vollständig zu ersetzen, so steht diese Ergänzung erst recht nicht zur Disposition. Wir müssen uns also nach weiteren Alternativen umsehen.

Die normale Objektquantifikation ist zwar dazu geeignet, unendliche Konjunktionen und Disjunktionen in einem Satz zusammenzufassen. Wir kommen aber nicht umhin, sie durch ein Prädikat zu ergänzen. Um darauf verzichten zu können, bräuchten wir eine Form der Quantifikation, die das letzte Vorkommen von , , $p^{“}$ in (27) nicht als singulären Terminus, sondern als Satz behandeln. Es reicht hier offensichtlich nicht aus, eine Gegenstandsvariable einfach durch einen Satz oder genauer: durch den Namen eines Satzes zu ersetzen. Wir müssen den Satz auch als solchen verwenden. Anders gesagt müsste die Quantifikation an dieser Stelle tatsächlich in Satzpositionen hinein erfolgen. Eine derart eigenwillige,

332 Es ist $\mathrm{zu}$ beachten, dass es sich hier um Instantiierungen von (27) in ihrer unvollständigen Form handelt und nicht um Einzelfälle einer (unendlichen) Liste von Sätzen oder Propositionen, über die regulär quantifiziert wird. Letztere sind grammatisch unbedenklich, erstere nicht. 
hybride Lesart, in der die verschiedenen Vorkommen der Variablen einmal für Gegenstände und einmal für Sätze stehen, gibt es jedoch nicht. ${ }^{333}$

\subsection{Substitutionelle Quantifikation}

Wir können das Problem allerdings so angehen, dass wir auf eine prinzipiell andere Interpretation zurückzugreifen. Hierfür kommt die substitutionelle Quantifikation in Frage. ${ }^{334}$ Ihre Besonderheit ist die, dass sie Variablen nicht mit Gegenstandsbereichen, sondern mit Substitutionsklassen verknüpft. Eine Variable ist hier nicht Platzhalter für einen Bereich, der typischerweise aus außersprachlichen Gegenständen besteht. Vielmehr steht sie für eine Klasse syntaktisch geeigneter sprachlicher Ausdrücke, kurz gesagt: für Namen. Wir begeben uns bei dieser Interpretation also auf eine übergeordnete Ebene des sprachlichen Bezugs. Quine bezeichnet dies auch als „,semantischen Aufstieg“. ${ }^{335}$ Etwas verkürzt kann man es als den Aufstieg von der Rede über die Welt zu der Rede über Sätze umschreiben. Diese metasprachliche Art der Quantifikation hat den Vorteil, dass die jeweiligen Substitutionsinstanzen grammatisch gesehen unproblematisch sind. Anders als bei der objektualen Lesart erfolgt die Quantifikation hier wirklich in Satzpositionen hinein. Daher müssen wir uns um ihre Wohlgeformtheit keine Sorgen machen.

Nun ist es aber eine Sache zu behaupten, um (25) mit den Mitteln der Logik darzustellen, müssen wir (27) einfach als eine substitutionelle Quantifikation auffassen. Eine andere ist es, sich auch den Sinn dieser Lesart verständlich zu machen. Logisch gesehen mag sie vielleicht ohne weiteres möglich sein. In der natürlichen Sprache fehlen uns jedoch die Mittel, um diese Art der Quantifikation auszudrücken. ${ }^{336}$ Wir quantifizieren nie über Namen und sagen Dinge wie: „Für alle Namen gilt, wenn ein Name diesen oder jenen Satz oder Gedanken bezeichnet, dann ist der Satz oder Gedanke soundso.“ In der natürlichen Sprache quantifizieren wir über Objekte. Eine gängige Art und Weise, die substitutionelle

\footnotetext{
${ }^{333}$ Vgl. Quine (1970), 11 f. u. 91 f.

334 Zur Unterscheidung von substitutioneller und objektualer bzw. referentieller Quantifikation vgl. Quine (1969), 63 ff. u. 104 ff.

${ }^{335}$ Vgl. Quine (1960), 270 ff. u. Quine (1970), 11.

${ }^{336}$ Vgl. Horwich (1998b), 4 Anm. 1 u. 124 f.
} 
Quantifikation verständlich zu machen, besteht daher auch darin, sie mit Hilfe syntaktischer und semantischer Ausdrücke und der objektualen Quantifikation zu paraphrasieren. ${ }^{337}$ Bei dieser Vorgehensweise quantifizieren wir also wiederum objektual, in diesem Fall über Substitutionsinstanzen substitutionell quantifizierender Aussagen. Wir erhalten dann eine Reformulierung unserer ursprünglichen Generalisierung folgender Art:

(31) Jede Substitutionsinstanz für „,p“ in dem Schema „Wenn Hannah über Karl sagt, dass $p$, dann $p$." ist wahr.

So gesehen besteht der Sinn der substitutionellen Quantifikation darin, die Wahrheit jeder formal gültigen Substitutionsinstanz zu behaupten. Wenn man es sich auf diese Weise verdeutlicht, kommt man also auch bei dieser Art von Quantifikation nicht um die Verwendung des Wahrheitsprädikates herum.

Zugegeben, dies ist nur eine Verdeutlichung des Sinns der substitutionellen Quantifikation mit den Mitteln der natürlichen Sprache. Prinzipiell ist nicht ausgeschlossen, dass sich diese Form der Quantifikation auch auf eine Weise verständlich machen lässt, die tatsächlich auf den Wahrheitsbegriff verzichtet. ${ }^{338}$ Es spricht auch grundsätzlich nichts dagegen, die natürliche Sprache um zusätzliche Mittel zu bereichern. Schließlich kommt es nicht selten vor, dass wir auf einer übergeordneten Ebene der Reflexion Themen und Probleme verhandeln, die im Bereich der natürlichen Sprache aufgekommen sind. Die substitutionelle Quantifikation wäre aber in jedem Fall eine überaus sperrige, wenig praktikable Bereicherung. Was die Einfachheit der Funktionsweise angeht, könnte sie es mit dem Wahrheitsprädikat nicht aufnehmen.

Mit der allgemeinen Beschaffenheit und Verständlichkeit des Begriffs verbindet sich aber nur ein Teil der Schwierigkeiten, die mit der substitutionellen Quantifikation einhergehen. Darüber hinaus gibt es mindestens zwei weitere, ernsthafte Bedenken, was diesen Versuch angeht,

${ }^{337}$ Vgl. Quine (1969), $106 \mathrm{f}$.

${ }^{338}$ Vgl. z. B. Hill (2002), insbes. 17 ff. 
das Wahrheitsprädikat zu ersetzen oder zu erklären. ${ }^{339}$ Zum einen ist die Reichweite der jeweiligen Substitutionsklasse prinzipiell auf die Sprache beschränkt, in der die Quantifikation formuliert ist. In diesem Fall wäre es das Deutsche. Wenn man bedenkt, dass es Gedanken oder Propositionen gibt, die sich in dieser Sprache nicht ausdrücken lassen, dann muss insbesondere die universelle Quantifikation als potentiell unvollständig erscheinen. Daraus ergibt sich zum anderen, dass manches zu verschiedenen Zeitpunkten durch unterschiedliche Sätze ausgedrückt wird. Auch dieser Umstand macht die Verwendung der substitutionellen Quantifikation kompliziert, denn auch er verlangt nach zusätzlichen Ergänzungen. Das macht es verständlich, wieso wir bei den fraglichen Generalisierungen und blinden Zuschreibungen das Wahrheitsprädikat verwenden und eben kein wie auch immer geartetes logisches Konstrukt.

Die substitutionelle Quantifikation verwirrt also mehr als sie erklären kann. Bei der objektualen Quantifikation dagegen liegen die Dinge anders. Ihre Funktionsweise ist uns intuitiv vertraut. Wenn sie sich auch nicht dazu eignet, das Wahrheitsprädikat zu ersetzen, so können wir es mit ihrer Hilfe zumindest verständlich machen. Wie sich nämliche ohne große Schwierigkeiten zeigen lässt, fügt sich diese Art der Quantifikation passgenau in das deflationistische Bild, welches ich zuvor zur Erklärung des Wahrheitsbegriffes angefertigt habe. Kern dieser Erklärung war das erwähnte Schema (ÄS). Wenn wir dies nun auf die entsprechende unendliche Konjunktion oder Disjunktion anwenden, dann ergibt sich die objektuale Standardquantifikation quasi von selbst. ${ }^{340}$

Die alltagssprachliche Generalisierung „Alles, was Hannah über Karl sagt, ist wahr." bringt mit wenigen Worten indirekt das zum Ausdruck, was als unendliche Konjunktion eigentlich unausdrückbar ist. Da die einzelnen Konjunkte jedoch alle die gleiche Form aufweisen, lassen sie sich alternativ in einem Schema zusammenfassen und durch eine Quantifikation verallgemeinern. Die Schwierigkeit liegt allerdings darin, dass sich hier nicht automatisch ein wohlgeformter Satz ergibt. Um zu einer ordentlichen objektualen Quantifikation der Art

(32) Für alle $p$, wenn Hannah über Karl sagt, dass $p$, dann ist $p$ wahr.

\footnotetext{
${ }^{339} \mathrm{Zu}$ den beiden folgenden Punkte vgl. Soames (1997), $7 \mathrm{ff}$.

${ }^{340} \mathrm{Vgl}$. auch für das Folgende Horwich (1998b), 31 ff.
} 
zu gelangen, ist ein Zwischenschritt nötig. Er besteht darin, das letzte Vorkommen von , $p$ “ durch das Wahrheitsprädikat zu ergänzen. Und das wiederum erreichen wir dadurch, dass wir das Schema (̈̈S) auf (25) anwenden. Dann nämlich lässt sich folgendes ableiten:

(33) Wenn Hannah über Karl sagt, dass er Tiramisu liebt, dann ist es wahr, dass er Tiramisu liebt, und wenn Hannah über Karl sagt, dass er schnarcht, dann ist es wahr, dass er schnarcht, und wenn Hannah über Karl sagt, dass er täglich joggen geht, dann ist es wahr, dass er täglich joggen geht ... usw. ${ }^{341}$

Dies ist gleichbedeutend mit der ursprünglichen Formulierung der Konjunktion in (25) und lässt sich wiederum auf folgendes Schema reduzieren:

(34) Wenn Hannah über Karl sagt, dass $p$, dann ist es wahr, dass $p$.

Und dies kann man nun ohne weiteres durch die fragliche Standardquantifikation verallgemeinern. Das Resultat ist der grammatisch sinnvolle quantifizierende Satz (32) einige Zeilen weiter oben.

Es lässt sich also festhalten, dass das Wahrheitsprädikat zwar in bestimmten sprachlichen Zusammenhängen redundant ist. Das gilt aber nur insofern, als durch das Prädizieren material äquivalente Sätze entstehen. Und es ist nur dort der Fall, wo Wahrheit unmittelbar gegebenen Sätzen oder Behauptungen zugeschrieben wird. Wo dies nicht so ist, führt der Verzicht auf das Prädikat $\mathrm{zu}$ gravierenden grammatischen Unstimmigkeiten beziehungsweise $\mathrm{zu}$ erheblicher theoretischer Reformulierungs- und Erklärungsarbeit. Denn prinzipiell lassen sich Generalisierungen und blinde Zuschreibungen zwar auch in Form von Quantifikationen ausdrücken. Bei Licht betrachtet ist dies aber keine wirkliche Alternative. In der objektualen Standardinterpretation ist das Wahrheitsprädikat nicht überflüssig, sondern ein unverzichtbarer Bestandteil. Der Rückgriff auf die substitutionelle Interpretation erscheint als die bessere Lösung. Denn die grammatisch sinnvolle Reformulierung

341 Anstelle von ,... ist es wahr, dass $p .$. “ kann man auch setzen ,... ist die Proposition, dass $p$, wahr ...“ bzw. „... ist der Satz ,p’ wahr ...“. Ich überlasse es dem Leser, die richtige Wahl zu treffen. Für die Argumentation dieses Kapitels ist dies nicht von Belang. 
unendlicher Konjunktionen und Disjunktionen kommt hier ohne das Wahrheitsprädikat aus. Was jedoch zunächst wie ein explanatorischer Vorteil aussieht, entpuppt sich schnell als das Gegenteil. Zum einen ist der genaue Sinn dieser Art Quantifikation intuitiv nicht zugänglich. In der natürlichen Sprache fehlen uns die Mittel sie auszudrücken. Zum anderen lässt sich der Begriff der substitutionellen Quantifikation nicht ohne komplizierte, theoretische Ergänzungen einführen. Die objektuale Lesart ist der substitutionellen daher letztlich überlegen.

Wenn man sich die Zusammenhänge und Hintergründe in dieser Deutlichkeit vor Augen führt, tritt unweigerlich die tragende Bedeutung des Schemas (ÄS) bei der Untersuchung des Wahrheitsbegriffs in den Blick. Es zeigt nicht nur die Redundanz einiger Verwendungsweisen. Geht es um das Verständnis der spezifischen Funktion des Wahrheitsprädikates, so ist dieses Schema überaus informativ. Wie gesehen ist es ein zentraler Bestandteil des sprachlich-logischen Mechanismus, der hinter den charakteristischen Verwendungsweisen des Wahrheitsbegriffs steckt. Der Umstand, dass es sich bei den Instantiierungen des Schemas um materiale Äquivalenzen handelt, macht es möglich, eine Behauptung performativ zu akzentuieren. So wird das Wahrheitsprädikat $\mathrm{zu}$ einem wertvollen rhetorischen Mittel der ausdrücklichen Zustimmung, Wertschätzung oder Empfehlung. Vor allem aber was Generalisierungen und indirekte Behauptungen betrifft, bildet das Schema den funktionalen Kern. Um zu sehen, dass eine allgemeine Aussage wie „Alles, was Hannah über Karl sagt, ist wahr." nichts anderes ist als die alltagssprachliche Kurzform einer gewöhnlichen Objektquantifikation, bedarf es keiner weiteren theoretischen Mittel. Wir müssen die Standardmittel der Logik lediglich um dieses Schema ergänzen. ${ }^{342}$

Somit erweist sich das deflationistische Wahrheitsverständnis als tragfähiger, als es auf den ersten Blick erscheinen mochte. Es bietet zwar keine explizite, reduktionistische Definition. Mit der Wahrheit verhält es

\footnotetext{
$342 \mathrm{Zu}$ dem Einwand, dass die Instanzen der Generalisierungen, die mit Hilfe des Wahrheitsprädikats formuliert werden, nicht das Gleiche aussagen wie die Generalisierungen selbst, vgl. Gupta (1993), 62 f. u. 67. Zu der Auffassung, dass das material aufgefasste Äquivalenzschema ( ̈̈S) die Ableitung einer Generalisierung durchaus legitimiert, vgl. aber Horwich (1998b), 124 u. 137. Eine materiale Inferenzregel lizenziert dann eine solche Ableitung. Vgl. Kap. 5.8 ff.
} 
sich wie mit vielen anderen logischen Begriffen. So wie sich zu Wesen und Natur der Negation oder der materialen Implikation nichts sagen lässt, so auch hier. Dagegen sind Auskünfte darüber, was es heißt, den Wahrheitsbegriff zu verwenden, durchaus möglich. Dann lassen sich die Instanzen von (ÄS) als die Axiome verstehen, die unserem Gebrauch zugrunde liegen. Durch unsere Bereitschaft, sie blind zu akzeptieren, legen wir fest, was der Ausdruck ,wahr“ bedeutet. Jemand verfügt also erst dann über den Wahrheitsbegriff, wenn er bereit ist, sich auf sämtliche unkontroversen Instanzen dieses Schemas festzulegen. Da dies aber unendlich viele sein können, lässt sich die deflationistische Wahrheitstheorie nicht abschließend und ausdrücklich formulieren. Vielmehr ergibt sich aus dem Gebrauch des Wahrheitsprädikates und der bedingungslosen Akzeptanz des Schemas (ÄS) eine Axiomatisierung oder implizite Definition. ${ }^{343}$

Wahrheit ist somit ein grundlegender, axiomatisierter Begriff. Mit dem Äquivalenzschema ( $\ddot{\mathrm{S} S}$ ) ist alles gesagt, was es dazu sagen gibt. Sicher, es gibt Sätze, die außerhalb dessen liegen, was die vorliegende Erklärung behandelt hat. Dazu gehören Beispiele wie: „Wahrheit ist das Ziel der Wissenschaft.“, „Die Wahrheit ist eine, doch der Meinungen sind viele.“ oder „In Gott ist Wahrheit.“ Für Aussagen dieser Art kann das deflationistische Erklärungsmodell nichts anbieten. Das ist aber kein Mangel dieses Modells. Vielmehr ist es ein Hinweis darauf, dass hier ein philosophischer Irrtum vorliegt, der aus einem grammatischen Missverständnis resultiert. Der Fehler besteht darin, von der grammatischen Oberflächenbeschaffenheit des Ausdrucks „wahr“ oder eines seiner alltagssprachlichen Äquivalente auf eine gewöhnliche Substanz zu schließen.

Dass (ÄS) der allgemeine Standard ist, den alle Vorkommnisse des Wahrheitsprädikates erfüllen müssen, unterstreicht noch einmal die These, wonach Überzeugungen insofern normativ signifikant sind, als sich mit ihnen immer ein Wahrheitsanspruch verbindet. Denn in der Rede vom Fürwahrhalten spiegelt sich der Umstand, dass Überzeugungen als Behauptungen mit dem Anspruch, wahr zu sein, geäußert und zugeschrieben werden. Diese Einsicht lässt sich nun mit Hilfe des Schemas

${ }^{343}$ Vgl. Leeds (1978), 122 u. Horwich (1998b), 31, 34 u. 121. 
(苂) bekräftigen. Zwar lesen wir es vor allem in der Richtung, wonach „Es ist wahr, dass $p^{\prime \prime}$ mit , $p$ “ äquivalent ist. Die Äquivalenz gilt aber ebenso gut in die andere Richtung. Demnach läuft jede Behauptung von , $p$ “ letztlich auch auf die Behauptung „Es ist wahr, dass $p$.“ hinaus. Man kann also aufgrund des Äquivalenzschemas zu der Feststellung kommen, dass das Wahrheitsprädikat zumindest implizit allgegenwärtig ist. ${ }^{344}$ In dieser umgekehrten Lesart lässt es sich als immanenter Bestandteil des Habens und Äußerns von Überzeugungen erkennen. Jede Behauptung enthält implizit als Anspruch, was sich durch das Wahrheitsprädikat explizit machen lässt.

Zusammenfassend lässt sich sagen, dass der Deflationismus Wahrheit auf der Grundlage materialer Äquivalenz erklärt. Die Idee ist, dass ein deklarativer Satz, dem Wahrheit zuerkannt wird, die gleiche Bedeutung hat wie sein Pendant ohne diese Zuerkennung. Es handelt sich also um eine semantische Konzeption von Wahrheit. Die Erklärung von Wahrheit geht zurück auf den Begriff der Bedeutung. Würde man nun Bedeutung wiederum mit Hilfe der Wahrheitsbedingungen eines Satzes oder einer Behauptung erklären, so würde man sich in einem explanatorischen Kreis bewegen. ${ }^{345}$ Will man keinen der beiden Begriffe aufgeben, so muss man sich für eine Erklärungsrichtung entscheiden. Diese Entscheidung habe ich bereits in den vorausgehenden Kapiteln getroffen, wo ich den Begriff der Bedeutung in Form einer inferentialistischen Gebrauchstheorie eingeführt und erläutert habe. ${ }^{346}$ Der semantische Inferentialismus versteht die Bedeutung einer Behauptung allein aus ihrem inferentiellen Zusammenhang heraus. Der Wahrheitsbegriff spielt dabei keine explanatorische Rolle. Die Erklärungsreihenfolge, die ich hier also vorschlage, ist die, durch Ausführungen über die Struktur der Bedeutung zum Begriff der Wahrheit vorzustoßen und nicht umgekehrt. Eine zirkuläre Erklärung wird dadurch vermieden.

\footnotetext{
${ }^{344}$ Vgl. Rescher (1973), 7 Anm. 14.

${ }^{345}$ Diese Einsicht geht zurück auf M. Dummett. Vgl. Dummett (1958/59), 148 f. Den Grundgedanken einer wahrheitskonditionalen Bedeutungstheorie formuliert Wittgenstein im Tractatus. Vgl. Wittgenstein (1984e), 28 (4.024).

${ }^{346}$ Vgl. Kap. 3.3 u. 5. Zur genau entgegengesetzten Auffassung, dass Wahrheit ein zentraler undefinierter Begriff ist, mit dessen Hilfe sich Bedeutung bestimmen lässt, vgl. Davidson (1984), XIV u. 24.
} 
In den verbleibenden Abschnitten werde ich eine abschließende Einschätzung von Funktion und Nutzen des Wahrheitsprädikates vornehmen. Es soll dabei um die noch offene Frage gehen, welchen Beitrag der Wahrheitsbegriff bei der Standardanalyse von Wissen generell, insbesondere aber bei deren normativ-pragmatischer Rekonstruktion leisten kann. Nach den letzten Abschnitten steht fest, dass es keine substantielle Theorie gibt, die die Wahrheitsbedingung über die charakteristische Funktions- und Verwendungsweise des Begriffs hinaus erklären kann. Dies werde ich nun dahingehend präzisieren, dass Wahrheit zwar ein rechtmäßiger Bestandteil der Standarddefinition von Wissen ist, zur tatsächlichen Erklärung des Begriffs aber nichts beiträgt.

\subsection{Die epistemologische Neutralität des Wahrheitsbegriffs}

Als sprachliches Mittel ist das Wahrheitsprädikat in Alltag und Wissenschaft hilfreich und nützlich. Es stattet uns mit expressiven Ressourcen aus, die zum Teil unersetzbar sind. Das gilt nicht unbedingt für den Einsatz als rhetorisches Mittel. Wir können zwar eine Festlegung performativ explizit machen, indem wir die entsprechende Behauptung unter Hinzunahme des Wahrheitsprädikates wiederholen. Und tatsächlich kommt es häufig genau auf diese Weise zum Einsatz. Wie aber die angeführten Beispiele zeigen, gibt es hier auch Alternativen. Das Wahrheitsprädikat ist als rhetorisches Mittel in bestimmten Gesprächssituationen typisch, aber nicht einzigartig.

Ohne eine wirkliche Alternative ist es dagegen, wenn es um die Formulierung von Generalisierungen geht. Das Wahrheitsprädikat versetzt uns in die Lage, uns indirekt auf bestimmte oder auch unendlich viele Behauptungen festzulegen. Obwohl sie uns nicht konkret oder in ihrer Gesamtheit gegeben sind, können wir die Behauptungen anderer blind und auf einen Schlag übernehmen und so unsere Zustimmung, Wertschätzung oder Empfehlung zum Ausdruck bringen. Dass das nützlich ist, habe ich oben an dem Beispiel „Alles, was Hannah über Karl sagt, ist wahr.“ veranschaulicht. Mit Hilfe des Wahrheitsprädikates kommt man darum herum, eine unendliche Liste all dessen zu erstellen, was Hannah über Karl sagt. Gleichzeitig lässt sich mit dieser Generalisierung jede Behauptung rechtfertigen, die Hannah über Karl macht. Angenommen jemand legt sich 
darauf fest, dass Karl Tiramisu liebt. Dass er dazu auch berechtigt ist, kann er dann damit begründen, dass Hannah über Karl sagt, dass er Tiramisu liebt und dass alles, was Hannah über Karl sagt, wahr ist.

Das Folgende gibt ein weiteres Beispiel für die generalisierende Funktion des Wahrheitsprädikates und den Nutzen, der sich daraus ziehen lässt. Nehmen wir an, ein Freund sagt zu mir nach einem gemeinsam besuchten Vortrag: „Das war zwar ein unterhaltsamer Vortrag. Aber nur das, was der Redner zu Beginn gesagt hat, ist wahr." Ich habe großes Vertrauen in das Urteil meines Freundes. Bisher hat er stets Recht behalten. Allerdings habe ich den Beginn des Vortrags verpasst. Ich habe nicht mitbekommen, was der Redner dort gesagt hat. Trotzdem kann ich ihm zustimmen, indem ich die Behauptung meines Freundes gleichsam blind übernehme und sage: „Was der Redner zu Beginn seines Vortrags gesagt hat, ist wahr."

Doch damit nicht genug. Zum Funktionsumfang des Wahrheitsprädikates gehört es auch, Schlussfolgerungen aus indirekten oder blinden Zuschreibungen $\mathrm{zu}$ ermöglichen. Angenommen ich lese einige Zeit nach dem besagten Vortrag in einer Besprechung, dass der Redner zu Beginn behauptet hat, es habe nie zuvor in der Geschichte der Menschheit weniger Gewalt gegeben als heute. Daraus kann ich nun schließen, dass es tatsächlich nie zuvor in der Geschichte der Menschheit weniger Gewalt gab als heute. Der Schluss von der indirekten Behauptung meines Freundes und der Information aus der Besprechung in der Zeitung auf diese konkrete Behauptung ist intuitiv plausibel. Er lässt sich aber auch als einen Beweis der klassischen Logik formulieren: ${ }^{347}$

(1) $x$ ist wahr

[Annahme]

(2) $x=$ die Proposition, dass $p$ [Annahme]

(3) Die Proposition, dass $p$, ist wahr [aus (1), (2)]

(4) Die Proposition, dass $p$, ist wahr $\supset p$

(5) $p$

[aus (3), (4)]

Das Wahrheitsprädikat spielt dabei eine tragende Rolle. Da jede beliebige Instanz von (ÄS) ohne weitere Vorbedingungen gültig ist, dürfen wir es als Axiom zu dem Beweis hinzunehmen. So erhalten wir ein Konditional, das

${ }^{347}$ Vgl. Künne (2003), 321. 
wir an entscheidender Stelle einfügen können. Durch die formale Rekonstruktion wird dann deutlich, warum der Schluss von (1) und (2) auf (5) tatsächlich auch formal gültig ist. Ebenfalls deutlich wird, dass (ÄS) letztlich auch für dieses Funktionsmerkmal des Wahrheitsprädikats verantwortlich ist. Denn dass wir von einer Generalisierung wieder zurück auf eine konkrete Instantiierung schließen können, verdanken wir ebenfalls seiner umfassenden Gültigkeit.

In der Philosophie ist all dies ebenfalls ausgesprochen nützlich. So erlaubt es die Generalisierungsfunktion des Wahrheitsprädikats beispielsweise, philosophische Positionen zuzuschreiben und zu verhandeln, ohne dass sie selbst vertreten werden müssen. Dies lässt sich an der Diskussion des metaphysischen Realismus eindrücklich veranschaulichen. ${ }^{348}$ In Kapitel 6.2 war von dieser Position schon einmal die Rede. Dort habe ich sie als die Auffassung charakterisiert, dass es Dinge in der Welt gibt, deren Existenz und Beschaffenheit unabhängig davon sind, ob und wie wir davon erfahren. Ein Realist könnte also beispielsweise folgendes behaupten:

(35) Es gibt Higgs-Bosonen, aber wir werden nie (ausreichend) Gründe dafür finden, dass es so ist.

Es ist aber natürlich auch möglich, Realist zu sein, ohne an die Existenz von Higgs-Bosonen zu glauben. Oder jemand ist Realist, aber diesbezüglich unentschieden. Irgendeine Überzeugung dieser Art wird er jedoch haben. In einer ausreichend langen Liste typischer Thesen gibt es sicherlich eine, die zu vertreten er bereit ist und die ihn als Realisten $\mathrm{zu}$ erkennen gibt. Eine solche Liste könnte man nun versuchen anzufertigen:

(36) Entweder es gibt Higgs-Bosonen, aber wir werden nie (ausreichend) Gründe dafür finden, dass es so ist, oder es gibt Strings, aber wir werden nie (ausreichend) Gründe dafür finden, dass es so ist, oder es gibt die Schleifen-Quantengravitation, aber wir werden nicht (ausreichend) Gründe dafür finden, dass es so ist, oder ...

${ }^{348}$ Vgl. Soames (1984), 413 f., Davidson (1990), 309 u. Field (2001), 120 f. 
Für ,..." ließe sich jeder erdenkliche Satz einfügen, der den obigen Beispielen entspricht. Um sicher zu gehen, müsste diese Liste jedoch unendlich lang sein - ein nicht besonders vielversprechendes Unterfangen. Es muss eine bessere Methode geben, um die Position des Realisten zu beschreiben. Und tatsächlich schafft hier das Wahrheitsprädikat Abhilfe. Denn wie wir ja wissen, können wir mit seiner Hilfe unendliche Konjunktionen und Disjunktionen in einem einzigen Satz zusammenfassen. Mit der folgenden allgemeinen Aussage gelingt es, die Position des Realisten auszudrücken, ohne zu wissen, was er im Einzelnen vertritt:

(37) Für mindestens ein $p, p$ ist wahr, aber wir werden nie (ausreichend) Gründe dafür finden, dass es so ist. ${ }^{349}$

Mit dieser quantifizierenden Aussage wird weder die Position des Realisten selbst noch eine mögliche Instantiierung dieser Generalisierung vertreten. Das Wahrheitsprädikat wird wiederum nur in seiner generalisierenden Funktion verwendet. Es fasst all das zusammen, was der Realist möglicherweise vertritt und verweist damit indirekt auf seine spezifisch realistische Position. Da auch hier wieder über Gegenstände (Propositionen, Sätze) quantifiziert wird, ist das Prädikat unverzichtbar. Denn mit einem unprädizierten Vorkommen von , $p^{“}$ wäre die existentielle Quantifikation kein grammatisch vollständiger Satz.

Etwas Anderes ist es, wenn jemand das Wahrheitsprädikat dazu benutzt, eine bestimmte realistische These zu bekräftigen. Dies geschieht beispielsweise, wenn er sagt:

(38) Es ist wahr, dass es Higgs-Bosonen gibt, obwohl wir nie (ausreichend) Gründe dafür finden werden, dass es so ist.

${ }^{349}$ Eine alternative Formulierung wäre:

(37') Jede Substitutionsinstanz für ,,$p^{\prime \prime}$ in dem Schema , ,p, aber wir werden nie (ausreichend) Gründe dafür finden, dass es so ist." ist wahr.

Hierbei handelt es sich jedoch wieder um eine objektuale Paraphrase der substitutionellen Quantifikation, die ich zuvor als problematisch eingestuft habe. 
In diesem Fall dient das Prädikat einem Sprecher als rhetorisches Mittel, um sich selbst explizit auf diese eine realistische These festzulegen. Auf dieselbe Art und Weise könnte man sich aber auch auf eine antirealistische These oder etwas ganz anderes festlegen. Anders also als es die Korrespondenztheorie vermuten lässt, ist Wahrheit selbst nicht notwendigerweise Thema der Diskussion um den metaphysischen Realismus. Nach dem deflationistischen Verständnis ist sie nur ein Mittel, um diese oder auch damit konkurrierende Positionen darzustellen.

Was also die Frage nach der Realität und ihrer Beschaffenheit betrifft, ist das Wahrheitsprädikat selbst vollkommen neutral. Und, so lässt sich anfügen, das gilt überhaupt für alle metaphysischen und epistemologischen Fragen. Diese Einschätzung lässt sich mit Alfred Tarski stützen, wenn er schreibt:

[W]e may accept the semantic conception of truth without giving up any epistemological attitude we may have had; we may remain naive realists, critical realists or idealists, empiricists or metaphysicians-whatever we were before. The semantic conception is completely neutral toward all these issues. ${ }^{350}$

Tarskis Überlegungen weichen von denen dieser Arbeit in einigen zentralen Punkten ab. Ziel seiner Untersuchung ist eine Definition des Wahrheitsbegriffs für formale Sprachen. Weder was diesen Definitionsversuch noch was die Einschränkung auf formale Sprachen betrifft, entspricht dies dem Ansatz dieser Arbeit. Allerdings geht auch er bei der Definition von Wahrheit von einer Form des Äquivalenzschemas (̈̈S) aus. ${ }^{351}$ Daher ist es nicht verwunderlich, dass auch er zu der Überzeugung kommt, dass das Wahrheitsprädikat in den genannten philosophischen Fragen keine Erklärungsarbeit leistet.

Das gilt nun auch für den Versuch, den Begriff des propositionalen Wissens zu definieren. Auch hier hat der Wahrheitsbegriff keinerlei Erklärungsfunktion. Zwar ist Wahrheit in den allermeisten Vorschlägen einer Definition von Wissen eine notwendige Bedingung. Das allein macht sie aber noch nicht zu einem erkenntnistheoretisch gehaltvollen Begriff. Im Gegenteil ist es vielmehr so, dass sie auch hier lediglich eine logisch-

${ }^{350}$ Tarski (1944), 362.

${ }^{351}$ Vgl. erneut Anm. 324. 
expressive Funktion erfüllt. Bei genauem Hinsehen stellt sich die Standarddefinition von Wissen nämlich als ein weiteres Beispiel für die Verwendung des Wahrheitsprädikates in seiner generalisierenden Funktion heraus. Folgendes Schema entspricht dem etablierten, dreigliedrigen Verständnis von Wissen:

(W) $S$ weiß, dass $p$, genau dann, wenn $S$ glaubt, dass $p$, wenn $S$ darin gerechtfertigt ist zu glauben, dass $p$, und wenn es wahr ist, dass $p$.

Mit den Einsichten der letzten Abschnitte im Hinterkopf lässt sich dies nun wiederum als die schematisierte Zusammenfassung einer potentiell unendlichen Konjunktion begreifen. Schließlich ist die Definition ja genau das: ein Schema, das der Überprüfung mutmaßlicher Fälle von Wissen dient. Es stellt den invariablen Kern dessen dar, was alles im Einzelnen als Wissen gelten kann. Anders gesagt handelt es sich um eine Generalisierung einer unendlich langen Liste von Einzelfällen der folgenden Art:

(39) $S$ weiß, dass Paris die Hauptstadt von Frankreich ist, genau dann, wenn $S$ glaubt, dass Paris die Hauptstadt von Frankreich ist, wenn $S$ darin gerechtfertigt ist zu glauben, dass Paris die Hauptstadt von Frankreich ist, und wenn Paris die Hauptstadt von Frankreich ist.

$S$ weiß, dass Kant Königsberg zu Lebzeiten nie verlassen hat, genau dann, wenn $S$ glaubt, dass Kant Königsberg zu Lebzeiten nie verlassen hat, wenn $S$ darin gerechtfertigt ist zu glauben, dass Kant Königsberg zu Lebzeiten nie verlassen hat, und wenn Kant Königsberg zu Lebzeiten nie verlassen hat. $\ldots$

Sämtliche Aussagen dieser Art ergeben sich, wenn man in (W) an die Stelle von , $p$ “ einen deklarativen Satz einfügt und die Worte ,es wahr ist, dass" streicht. Denn für sich genommen lässt sich jede der einzelnen Aussagen, die durch die Generalisierung zusammengefasst werden, formulieren, ohne den Ausdruck „wahr“ zu verwenden. Insofern durch die Standarddefinition jedoch über diese Einzelaussagen quantifiziert wird, ist das Wahrheitsprädikat nötig. Denn auch hier gilt, dass mit einem unprädizierten Vorkommen von ,,$p^{\prime}$ noch kein grammatisch vollständiger Satz vorliegt. Das ist die Lehre, die sich aus der obigen Betrachtung der 
objektualen Quantifikation ziehen lässt: Ohne das Wahrheitsprädikat wäre die Standarddefinition grammatisch gesehen unvollständig.

Das Hinzufügen des Wahrheitsprädikates ist auch hier wieder durch (ÄS) legitimiert. Es garantiert, dass die Instanzen der Definition immer das Gleiche zum Ausdruck bringen, egal ob sie mit oder ohne das Wahrheitsprädikat formuliert werden. Ob ich nun sage:

(39) $S$ weiß, dass Paris die Hauptstadt von Frankreich ist, genau dann, (...) wenn Paris die Hauptstadt von Frankreich ist.

oder:

(39') $S$ weiß, dass Paris die Hauptstadt von Frankreich ist, genau dann, (...) wenn es wahr ist, dass Paris die Hauptstadt von Frankreich ist. ${ }^{352}$

macht hier keinen Unterschied. Der Wahrheitszusatz ändert nichts am Gehalt der jeweiligen Aussage. Auch hier sind , $p$ “ und „Es ist wahr, dass $p$." material äquivalent.

So gesehen ist Wahrheit keine notwendige Bedingung für Wissen, sondern allenfalls für seine Definition in der traditionellen, dreiteiligen Form. In der Standarddefinition hat das Prädikat „ist wahr“ die logischgrammatische Funktion, die Quantifikation über eine unendlich große Zahl von Einzelfällen zu ermöglichen. Für die Instantiierungen der Definition ist es dagegen überflüssig. Die jeweiligen Sätze sind auch so grammatisch vollständig. Ebenso wenig macht es inhaltlich einen Unterschied. Nach dem Modell der Standardanalyse bringt eine partikulare Wissenszuschreibung mit oder ohne Wahrheitsprädikat das Gleiche zum Ausdruck. Oder, etwas anders formuliert, sie beschreibt immer denselben Sachverhalt. Wahrheit ist also in Bezug auf Wissen deskriptiv gesehen verzichtbar. Wirklich notwendig sind hier nur Überzeugungen und deren Rechtfertigungen. Damit ist allerdings über die normativen Aspekte von Wissenszuschreibungen noch nichts gesagt. Wenn wir diese hinzunehmen, wird die erkenntnistheoretische Bedeutung des Wahrheitsbegriffes ersicht-

${ }^{352}$ Hier sind wieder Varianten möglich. Für meine Überlegungen ist jedoch nicht von Belang, ob man sagt: „... wenn die Proposition, dass Paris die Hauptstadt von Frankreich ist, wahr ist.“ oder: „... wenn der Satz ,Paris ist die Hauptstadt von Frankreich.' wahr ist.“ 
lich. Und so wird auch verständlich, was es mit der Wahrheitsbedingung im konkreten Einzelfall von Wissen auf sich hat.

\subsection{Wissen normativ verstanden}

Die einzelnen Gedanken, die ich in diesem Kapitel entwickelt habe, lassen sich nun abschließend zusammenführen. Eingangs habe ich angedeutet, wie sich der Wahrheitsbegriff in das zuvor entworfene Theoriemodell aus normativer Pragmatik und inferentieller Semantik einfügen lässt. Dort habe ich Wissen zwar gemäß dem dreiteiligen Standardmodell, aber von einem sozialpragmatischen Standpunkt aus charakterisiert. Dieser ist, so eine der Kernthesen dieser Arbeit, immer ein normativer Standpunkt. Demnach betrachten wir jemanden in der diskursiven Praxis als Wissenden, indem wir ihm eine Festlegung und eine Berechtigung zuschreiben und indem wir dieselbe Festlegung für uns selbst übernehmen. Dies habe ich dahingehend präzisiert, dass die Verwendung des Wahrheitsprädikates einem Akt der Zustimmung, Wertschätzung oder Empfehlung gleichkommt. In Fällen der direkten Wahrheitszuschreibung, ist dies die einzige Funktion dieses Prädikats. In Fällen der indirekten Wahrheitszuschreibung wird es durch die Generalisierungsfunktion ergänzt. Dadurch wird eine indirekte Zustimmung, Wertschätzung oder Empfehlung möglich. Dies lässt sich mit den Einsichten der letzten Abschnitte vertiefen. Danach hat das Wahrheitsprädikat zwar in der Standarddefinition von Wissen genau diese generalisierende Funktion, nicht jedoch in konkreten Einzelfällen von Wissen. Dort erfüllt es die normativ-expressive Funktion der direkten Zustimmung, Wertschätzung oder Empfehlung. Von einem normativen Standpunkt aus gesehen lässt sich dies wie folgt plausibilisieren.

Das deflationistische Verständnis von Wahrheit erlaubt es, bei Instantiierungen der Standarddefinition von Wissen auf das Wahrheitsprädikat zu verzichten. Denn deskriptiv gesehen wiederholt sich an der Position der Wahrheitsbedingung bloß das, was dem vermeintlich Wissenden bereits als Überzeugung zugeschrieben wird. In normativpragmatischer Hinsicht kommt dies aber einem Perspektivwechsel mit Blick auf diese Überzeugung gleich. Der Gehalt bleibt zwar derselbe, nicht aber die Person, die sich darauf festlegt. Zuvor habe ich den 
Sprachgebrauch prinzipiell als eine soziale Praxis charakterisiert. Ich habe geltend gemacht, dass die diskursive Praxis auf einer Ich-Du-Struktur basiert. ${ }^{353}$ Betrachtet man nun auch die Zuschreibung von Wissen von einer sozialpragmatischen Warte aus, dann ist diese Ich-Du-Struktur auch hier die Grundlage, auf der sich der besagte Perspektivwechsel vollzieht. Dann wird auch das wiederholte Vorkommen ein und derselben Überzeugung in den einzelnen Instanzen der traditionellen Wissensdefinition verständlich.

In den Ausführungen der vorausgehenden Kapitel kam Wahrheit lediglich als Anspruch vor. In normativ-pragmatischer Hinsicht entspricht dies einer Fokussierung des Ich-Teils der Ich-Du-Struktur. Als pragmatisches Pendant des Fürwahrhaltens ist der Sprechakt des Behauptens zunächst einmal das Erheben eines Wahrheitsanspruchs. Dadurch soll jemand anderes die fragliche Behauptung gleichermaßen für sich übernehmen können. Damit dieser Anspruch auch erfüllt werden kann, musste als nächstes gezeigt werden, dass und inwiefern es sich dabei um einen sinnvollen und bedeutsamen Sprechakt handelt. Nach dem Begriff der Überzeugung habe ich daher zunächst den der Bedeutung eingeführt. Daher steht dieser in der Erklärungsreihenfolge vor dem der Wahrheit. Das Spiel des Gebens und Verlangens von Gründen beziehungsweise von Festlegungen und Berechtigungen muss, sowohl was die Praxis als auch was die Theorie angeht, etabliert sein, bevor es um die Erklärung von Wahrheit gehen kann. Kurz gesagt kann etwas nur für den wahr oder falsch sein, für den es auch eine Bedeutung hat.

Die Begriffe der Festlegung und der Berechtigung bilden die Grundlage für die normativ-pragmatische Rekonstruktion der Überzeugungs- und Rechtfertigungsbedingung für Wissen. Dies ist zugleich die Grundlage für die Erklärung der Wahrheitsbedingung. Denn in normativ-pragmatischer Hinsicht haben Wahrheit und Rechtfertigung große Ähnlichkeit. Hier wie dort kommt der Du-Teil der Ich-Du-Struktur ins Spiel. Ein Sprecher verpflichtet sich mit einer Behauptung dazu, diese auf Nachfrage zu rechtfertigen. Gelingt ihm das, so betrachten andere ihn als zu dieser Behauptung berechtigt. Entsprechend verhält es sich mit dem Wahrheitsanspruch. Mit seiner Behauptung beansprucht ein Sprecher, dass

${ }^{353}$ Vgl. erneut Kap. 4.4. 
diese wahr ist. Andere können diesen Anspruch billigen, indem sie sich selbst ebenfalls auf diese Überzeugung festlegen. Somit ist für die Anerkennung des Wahrheitsanspruchs derselbe Perspektivwechsel notwendig, wie bei der Zuschreibung einer Berechtigung. Ein Sprecher legt sich auf eine Überzeugung fest. Er kann sich dazu aber nicht auch noch selbst berechtigen. Das können nur andere tun. Gleichermaßen kann er zwar mit seiner Festlegung einen Wahrheitsanspruch äußern. Er kann diesen aber nicht selbst erfüllen. Auch das müssen andere tun, und zwar indem sie die Festlegung für sich übernehmen.

Aus der Zuschreibung einer Festlegung und einer Berechtigung sowie der Anerkennung eines damit zusammenhängenden Wahrheitsanspruchs ergibt sich schließlich die Zuschreibung von Wissen. Die Erfüllung eines Wahrheitsanspruchs und die Zuschreibung von Wissen gehen also Hand in Hand. Wer den Wahrheitsanspruch eines anderen Sprechers anerkennt, übernimmt den normativen Status der Festlegung für sich selbst. Man kann auch sagen, dass er den anderen durch die Wissenszuschreibung zu seiner Festlegung autorisiert. Damit setzt sich fort, was in der sprachlichen Praxis ohnehin bereits in Gang ist. Schließlich ist die Grundlage des bedeutsamen, inferentiell gegliederten Sprachgebrauchs das Geben und Verlangen von Gründen. Das gegenseitige Rechtfertigen ist dort ein immanenter Bestandteil. Behauptungen sind Sprechakte, die berechtigt sein können oder auch nicht. Anders gesagt ist der Sprachgebrauch immer ein Spiel aus wechselseitiger Autorisierung und deren Verneinung.

All dies gilt nun auch für die Zuschreibung von Wissen. So wie eine Berechtigung eine Form der Anerkennung von Autorität ist, so ist es auch eine Wissenszuschreibung. Wissen ist also letztlich auch nichts anderes als ein normativer Status, dies allerdings nur auf synkategorematische Weise. ${ }^{354}$ Denn er entsteht erst durch das Zusammenspiel des Status der Festlegung und der Berechtigung. Er setzt sich zusammen aus einer Festlegung, die aus zwei unterschiedlichen sozialen Perspektiven vorgenommen wird, und einer Berechtigung. Aufgrund dieser Zusammengesetztheit ergibt sich für Wissen auch eine andere Form der Autorität als bei der Berechtigung. Wissen hat hier eine eigene Qualität.

354 Brandom bezeichnet Wissen auch als „hybriden deontischen Status“. Vgl. Brandom (1994), 202. 
Dabei geht es nicht mehr nur um die Konstitution von Rationalität und Bedeutung. Jemanden als Wissenden $\mathrm{zu}$ betrachten und $\mathrm{zu}$ behandeln bedeutet, ihm eine eigenständige Form von Autorität zuzusprechen. Man betrachtet ihn, wie Edward Craig es auch nennt, als einen ,guten Informanten“. ${ }^{355}$ Wer dem, was jemand behauptet, das Zertifikat „Wissen“ verleiht, vertraut ihm mehr, als wenn er ihm dazu einfach die Berechtigung erteilt. Das Vertrauen ist jetzt so groß, dass der andere selbst dazu bereit ist, die Verantwortung für diese Behauptung $\mathrm{zu}$ übernehmen. $\mathrm{Er}$ signalisiert, gegebenenfalls selbst ausreichend Gründe dafür angeben zu können.

Damit stellt sich das deflationistische Wahrheitsverständnis als eine ideale Ergänzung der normativ-pragmatischen Erklärung von Überzeugung und Rechfertigung heraus. Indem es Wahrheit als eine logisch-expressive Eigenschaft begreift, ist es ein Gewinn für das ursprüngliche Theoriemodell dieser Arbeit. Das macht es möglich, bei der Erklärung von Wissen auf Wahrheit als eine substantielle Eigenschaft zu verzichten. Mit Hilfe des deflationistischen Wahrheitsbegriffs ist eine vollständige Rekonstruktion der Standardanalyse von Wissen mit den Mitteln einer normativen Pragmatik und einer inferentiellen Semantik möglich. Wissen lässt sich auf die beiden normativen Status der Festlegung und der Berechtigung zurückführen. Im Zentrum der Überlegungen steht das, was wir tun, wenn wir jemand als Wissenden behandeln. Dabei spielt der Wahrheitsbegriff keine eigenständige erklärende Rolle. Anders als die gerechtfertigte Überzeugung ist Wahrheit für Wissen nicht konstitutiv. Wahrheit ist nur dann notwendig, wenn es darum geht, Fälle zu verallgemeinern, in denen gerechtfertigte Überzeugungen von anderen autorisiert werden. Wenn Wahrheit bei der Erklärung von Wissen relevant und unverzichtbar ist, dann in dieser logisch-expressiven Hinsicht. Ohne das Wahrheitsprädikat ließe sich Wissen nicht in Form der Standarddefinition verallgemeinern. Was wir aber tun, wenn wir jemand Wissen zuschreiben, ist nicht darauf angewiesen.

Somit lässt sich Tarskis Einschätzung bestätigen, dass Wahrheit epistemologisch gesehen ein neutraler Begriff ist. Zwar gehört die Wahrheitsbedingung zum Kernbestand des traditionellen Verständnisses

${ }^{355}$ Vgl. Craig (1993). 
von Wissen. Explanatorisch gesehen ist sie jedoch grundsätzlich irrelevant. Die anti-intellektualistische Grundintention dieser Arbeit, wonach Wissen eine Art Können ist, bleibt also unberührt. Die Rekonstruktion von Wissen durch die Kombination einer normativen Pragmatik mit einer inferentiellen Semantik lässt sich auch dadurch nicht in Zweifel ziehen, dass Wahrheit traditionell als eine notwendige Bedingung für Wissen gilt. Auch wenn Wahrheit ein notwendiger Bestandteil der Wissensdefinition ist, bleibt Können die Voraussetzung für Wissen. Ohne ein Beherrschen des regelgeleiteten und inferentiell gegliederten Sprachspiels der Überzeugung sind weder Wissen noch Definitionen möglich. 
